\title{
Electromagnetic field correlators and the Casimir effect for planar boundaries in AdS spacetime with application in braneworlds
}

\author{
A. A. Saharian๑, A. S. Kotanjyan, and H. G. Sargsyan® \\ Department of Physics, Yerevan State University, 1 Alex Manoogian Street, 0025 Yerevan, Armenia
}

(Received 17 September 2020; accepted 18 October 2020; published 11 November 2020)

\begin{abstract}
We evaluate the correlators for the vector potential and for the field strength tensor of the electromagnetic field in the geometry of two parallel planar plates in AdS spacetime. Two types of boundary conditions are considered on the plates. The first one is a generalization of the perfect conductor boundary condition and the second one corresponds to the confining boundary conditions. By using the expressions for the correlators, the vacuum expectation values (VEVs) of the photon condensate and of the electric and magnetic fields squared are investigated. As another important local characteristic of the vacuum state we consider the VEV of the energy-momentum tensor. The Casimir forces acting on the plates are decomposed into the self-action and interaction parts. It is shown that the interaction forces are attractive for both types of boundary conditions. At separations between the plates larger than the curvature radius of the background geometry they decay exponentially as functions of the proper distance. The self-action force per unit surface of a single plate does not depend on its location and depending on the boundary condition and on the number of spatial dimensions can be either attractive or repulsive with respect to the AdS boundary. By using the generalized zeta function technique we also evaluate the total Casimir energy. Applications are given in $Z_{2}$-symmetric braneworld models of the Randall-Sundrum type for vector fields with even and odd parities.
\end{abstract}

DOI: 10.1103/PhysRevD.102.105014

\section{INTRODUCTION}

In a large number of physical problems the interactions of quantum fields are expressed in terms of boundary conditions imposed on the field operator. This idealization essentially simplifies the quantization and renormalization procedures. For points outside of boundaries the structure of divergences is the same as that in the boundary-free theory and consequently the renormalization prescriptions for local physical observables (like the expectation values of the energy-momentum tensor and the current density) are the same as well. Additional divergences on constraining boundaries are removed by renormalization of the physical characteristics located on them. Depending on the specific problem, the physical nature of boundaries can be different. The examples include macroscopic bodies in quantum electrodynamics, interfaces separating different phases of the theory, horizons in gravitational physics, branes in higher-dimensional models and so on. Another type of constraints on the field operator, periodicity conditions, appear in field-theoretical models with compact

Published by the American Physical Society under the terms of the Creative Commons Attribution 4.0 International license. Further distribution of this work must maintain attribution to the author(s) and the published article's title, journal citation, and DOI. Funded by SCOAP . spatial dimensions. The boundary conditions on the field operator modify the spectrum of the field fluctuations and as a consequence the expectation values of physical quantities are shifted. This general class of phenomena is known as the Casimir effect (for reviews see [1]).

The physical characteristics in the Casimir effect depend on the field, on the bulk and boundary geometries and on the boundary conditions imposed. In particular, motivated by applications in gravity, cosmology and condensed matter systems, the investigations of the influence of the background geometry are of special interest. Already in the case of free fields closed analytic expressions for boundaryinduced contributions in the expectation values of physical observables are obtained for highly symmetric geometries. Particularly, the de Sitter and anti-de Sitter (AdS) spacetimes have attracted a great deal of attention. These spacetimes possess the same number of symmetries as the Minkowski spacetime and, as a consequence, a large number of physical problems are exactly solvable on their background. This gives an idea on the influence of gravitational field on physical phenomena in more complicated geometries.

In the present paper, as a background geometry we consider the AdS spacetime. In addition to high symmetry, the importance of this geometry in quantum field theory is motivated by a number of other reasons. The AdS spacetime is not globally hyperbolic and the early interest was 
mainly related to principal questions of the quantization procedure in such spacetimes. To yield well-defined dynamics, boundary conditions should be chosen on timelike conformal infinity and this brings several qualitatively new features compared to the Minkowskian theories. In particular, a new type of instabilities may arise. The importance of such studies is also due to the appearance of AdS spacetime as a ground state in extended supergravity and in string theories and as near horizon limit of extremal black holes and black strings. The nonzero curvature of the AdS spacetime provides an infrared regulator for correlation functions, consistent with supersymmetry and modular invariance [2]. Another new feature of the dynamics, that distinguishes the AdS bulk from the Minkowski one, is the existence of consistent theories for interacting higher spin fields. On top of all this, the geometrical properties of the AdS spacetime play a crucial role in two fascinating modern developments of highenergy physics. The first one, the AdS/CFT correspondence (see [3] for reviews), is a realization of the holographic principle. It states a duality between theories formulated in different numbers of spacetime dimensions: the supergravity or string theory in AdS bulk and conformal field theory on its boundary. Among the most important implications of this correspondence is the possibility for the investigation of nonperturbative effects in one theory through the weak coupling expansion of the dual theory. In addition to the high-energy physics, the recent developments include applications in condensed matter physics (holographic superconductors, quantum phase transitions, and topological insulators) [4]. The second focus of intense interest with AdS spacetime as the background geometry is various types of braneworld models [5] where the standard model fields are restricted to a hypersurface (brane) embedded in a higher-dimensional spacetime. Initially proposed for a resolution of the gauge hierarchy problem, these models give new insights into various problems of particle physics and cosmology. The existence of the branes on which the matter fields are confined is predicted also by string theories.

The boundary-induced quantum vacuum effects for planar branes have been widely studied in background of the AdS bulk for scalar [6,7], fermionic [8] and gauge $[9,10]$ fields. These investigations were mainly motivated by the possibility of the radion field stabilization in Randall-Sundrum-type braneworld models by using the Casimir forces acting on the branes, by generation of the cosmological constant on the branes and by extensions of the AdS/CFT correspondence to the case with boundaries in the conformal field theory side [11]. The vacuum expectation values of the energy-momentum tensor for scalar and fermion fields were investigated in [12]. The vacuum energy, the energy-momentum tensor, and the current density for charged fields in higher-dimensional models with compact subspaces have been considered in $[13,14]$. The models on the AdS bulk with de Sitter and AdS branes have been discussed in [15].

In the present paper we consider both the local and global effects for quantum electromagnetic field induced by two parallel plates in the AdS bulk with an arbitrary number of spatial dimensions. The propagators for vector fields in AdS spacetime in the absence of additional boundaries or branes have been considered in [16]. The different types of boundary conditions for vector fields on the AdS boundary and their interpretations in the context of AdS/CFT correspondence were considered in [17]. The dynamics of bulk gauge fields in the 5D Randall-Sundrum model have been discussed in [18]. The electromagnetic Casimir energy and the forces acting on the branes in 5D RandallSundrum model have been investigated in $[9,10]$ for the perfectly conducting boundary condition. For the same boundary condition, the two-point functions and the vacuum expectation value of the energy-momentum tensor in the geometry of a single plate on AdS bulk with general number of spatial dimensions were considered in [19-22]. The electromagnetic Casimir effect in de Sitter spacetime for planar boundaries has been discussed in $[23,24]$. The electromagnetic two-point functions and the Casimir effect in background of Friedmann-Robertson-Walker cosmologies with power-law scale factors were considered in [25].

The outline of the paper is as follows. In the next section we describe the geometry of the problem and present the mode functions. The two-point functions for the vector potential and field strength tensor are presented in Secs. III and IV, respectively. By using the two-point functions for the field strength tensor, the vacuum expectation values (VEVs) of the electric and magnetic fields squared and the photon condensate are investigated in Sec. V. The VEV of the energy-momentum tensor is discussed in Sec. VI. The Casimir forces acting on the plates are considered in Sec. VII. In Sec. VIII we investigate the total vacuum energy by using the zeta function regularization scheme. The applications of the obtained results to higher-dimensional generalizations of the Randall-Sundrum-type braneworld models are discussed in Sec. IX. The main results are summarized in Sec. X.

\section{BACKGROUND GEOMETRY AND THE MODES}

We consider a quantum electromagnetic field with the vector potential $A_{\mu}(x), \mu=0,1, \ldots, D$, and with the field strength tensor $F_{\mu \nu}=\partial_{\mu} A_{\nu}-\partial_{\nu} A_{\mu}$ in a $(D+1)$-dimensional spacetime with the metric tensor $g_{\mu \nu}$. The dynamics of the field is governed by the Maxwell equation

$$
\nabla_{\nu} F^{\mu \nu}=\frac{1}{\sqrt{|g|}} \partial_{\nu}\left(\sqrt{|g|} F^{\mu \nu}\right)=0,
$$

where $g=\operatorname{det}\left(g_{\mu \nu}\right)$. We will assume that the vector potential is constrained by the Lorentz condition 


$$
\nabla_{\mu} A^{\mu}=\frac{1}{\sqrt{|g|}} \partial_{\mu}\left(\sqrt{|g|} A^{\mu}\right)=0
$$

When boundaries are present, in order to have a well-posed Cauchy problem one needs to impose appropriate boundary conditions on the field.

In the present paper the background geometry is the AdS spacetime generated by a negative bulk cosmological constant $\Lambda$. In the Poincaré coordinates $\left(x^{0}=t, x^{1}, \ldots\right.$, $\left.x^{D-1}, x^{D}=z\right)$ the metric tensor is given by

$$
g_{\mu \nu}=\left(\frac{\alpha}{z}\right)^{2} \eta_{\mu \nu}
$$

where $\eta_{\mu \nu}=\operatorname{diag}(1,-1, \ldots,-1)$ is the metric tensor for $(D+1)$-dimensional Minkowski spacetime. For the coordinates one has $-\infty<x^{i}<+\infty$ for $i=0,1, \ldots, D-1$, and $0 \leq z<\infty$. The geometry is conformally related to the half of the Minkowski spacetime. The hypersurfaces $z=0$ and $z=\infty$ present the boundary and the horizon of the AdS spacetime. The parameter $\alpha$ is expressed in terms of the negative cosmological constant $\Lambda$ as $\alpha=$ $\sqrt{(1-D) D /(2 \Lambda)}$. Instead of the coordinate $z$ one can introduce the coordinate $y$ in accordance with $y=$ $\alpha \ln (z / \alpha),-\infty<y<+\infty$. In terms of this coordinate the metric is given by $g_{\mu \nu}=\operatorname{diag}\left(e^{-2 y / \alpha} \eta_{i k},-1\right)$ with $i, k=0,1, \ldots, D-1$. The gauge condition (2.2) does not fix the vector potential uniquely. We will impose an additional condition $A^{D}=0$ [for consistency with (2.2); see [10]]. Under this condition the constraint (2.2) is reduced to $\partial_{\mu} A^{\mu}=0$.

We are interested in the effects of two codimension one plates (branes in braneworld models), parallel to the AdS horizon, on the properties of the electromagnetic vacuum. The locations of the plates will be denoted by $z=z_{1}$ and $z=z_{2}, z_{2}>z_{1}$. If we denote by $y_{1}$ and $y_{2}$ the corresponding values of the $y$ coordinate, $y_{j}=\alpha \ln \left(z_{j} / \alpha\right), j=1,2$, then the proper distance between the plates is expressed as $a=y_{2}-y_{1}=\alpha \ln \left(z_{2} / z_{1}\right)$. Two types of boundary conditions will be considered below. The first one is the higher-dimensional generalization of the perfect conductor boundary condition in $3 D$ electrodynamics and reads

$$
n^{\mu_{1} *} F_{\mu_{1} \cdots \mu_{D-1}}=0, \quad z=z_{1}, z_{2},
$$

where ${ }^{*} F_{\mu_{1} \cdots \mu_{D-1}}=\varepsilon_{\mu \nu \mu_{1} \cdots \mu_{D-1}} F^{\mu \nu} /(D-1)$ ! is the dual of the field tensor and $n^{\mu}$ is the normal vector to the boundary. As the second type of boundary conditions we will take the condition

$$
n^{\mu} F_{\mu \nu}=0, \quad z=z_{1}, z_{2} .
$$

It is used in bag models of hadrons for the confinement of gluons inside the bag. Both the conditions (2.4) and (2.5) are gauge invariant.
In the problem under consideration, all the properties of the quantum vacuum are encoded in two-point functions or the vacuum fluctuation correlators. For the evaluation of the correlators we will use the mode-sum method. In that method the two-point functions are presented in the form of a sum over the products of the complete set of the electromagnetic modes obeying the boundary conditions. We will denote the complete set for the vector potential by $\left\{A_{(\beta) \mu}, A_{(\beta) \mu}^{*}\right\}$, where $\beta$ corresponds to the set of quantum numbers specifying the modes and the star stands for the complex conjugate. In accordance with the problem symmetry the dependence of the modes on the coordinates $x^{l}$, $l=0,1, \ldots, D-1$, can be taken in the form $e^{i k_{l} x^{l}}$ with the wave vector components $k_{l}$. With this choice and by taking into account that $A_{(\beta) D}=0$, the boundary condition (2.4) is reduced to $A_{(\beta) l}=0$ for $z=z_{1}, z_{2}$, and from the condition (2.5) we get $\partial_{D} A_{(\beta) l}=0, z=z_{1}, z_{2}$. This shows that in the geometry under consideration the conditions (2.4) and (2.5) are the analogs of Dirichlet and Neumann boundary conditions for scalar fields. The $z$ dependence of the modes can be found from the field equation and the mode functions for the vector potential are presented as

$A_{(\beta) \mu}(x)=\epsilon_{(\sigma) \mu} z^{D / 2-1}\left[c_{1} J_{D / 2-1}(\lambda z)+c_{2} Y_{D / 2-1}(\lambda z)\right] e^{i k_{l} x^{l}}$,

where $J_{\nu}(x)$ and $Y_{\nu}(x)$ are the Bessel and Neumann functions, $k_{0}=\omega=\sqrt{\lambda^{2}+k^{2}}$, and $k^{2}=\sum_{l=1}^{D-1} k_{l}^{2}$. The polarization vector $\epsilon_{(\sigma) \mu}$, with $\sigma=1, \ldots, D-1$ corresponding to different polarizations, is normalized by the condition $\eta^{\mu \rho} \epsilon_{(\sigma) \mu} \epsilon_{\left(\sigma^{\prime}\right) \rho}=-\delta_{\sigma \sigma^{\prime}}$. From the gauge conditions it follows that $\epsilon_{(\sigma) D}=0$ and $\eta^{\mu \rho} k_{\mu} \epsilon_{(\sigma) \rho}=0$. The set $\beta$ of quantum numbers is specified as $\beta=\sigma, \lambda, k_{1}, \ldots, k_{D-1}$. The normalization condition for the modes (2.6) has the form

$\int d^{D} x \sqrt{|g|}\left[A_{\left(\beta^{\prime}\right) \mu}^{*} \nabla^{0} A_{(\beta)}^{\mu}-\left(\nabla^{0} A_{\left(\beta^{\prime}\right) \mu}^{*}\right) A_{(\beta)}^{\mu}\right]=4 i \pi \delta_{\beta \beta^{\prime}}$,

where $\delta_{\beta \beta^{\prime}}$ is understood as the Kronecker delta for discrete components of $\beta$ and the Dirac delta function for the continuous ones. Note that for a scalar field with the curvature coupling parameter $\xi$ and the mass $m$ the radial part of the mode functions has the form $z^{D / 2}\left[c_{1} J_{\nu_{s}}(\lambda z)+\right.$ $\left.c_{2} Y_{\nu_{s}}(\lambda z)\right]$, where $\nu_{s}=\sqrt{D^{2} / 4-D(D+1) \xi+m^{2} \alpha^{2}}$. This shows that, unlike the case of the Minkowski bulk, for the AdS bulk the electromagnetic modes are not reduced to the set of massless scalar modes with minimal or conformal couplings.

The plates $z=z_{1}$ and $z=z_{2}$ divide the space into three parts: the region between the AdS boundary and plate $z=z_{1}, 0 \leq z \leq z_{1}$ (region I), the region between the plates, $z_{1} \leq z \leq z_{2}$ (region II), and the region between the plate $z=z_{2}$ and the horizon, $z_{2} \leq z<\infty$ (region III). 
The coefficients $c_{1}$ and $c_{2}$ in (2.6) depend on the region. We will consider the region between the plates. From the boundary condition on $z=z_{1}$ it is seen that

$$
c_{1}=C Y_{\nu}\left(\lambda z_{1}\right), \quad c_{2}=-C J_{\nu}\left(\lambda z_{1}\right),
$$

where

$$
\nu= \begin{cases}D / 2-1, & \text { for condition }(2.4), \\ D / 2-2, & \text { for condition }(2.5),\end{cases}
$$

and the constant $C$ is determined from the normalization condition (2.7). With the coefficients from (2.8), the mode functions in region II are expressed as

$$
A_{(\beta) \mu}=C \epsilon_{(\sigma) \mu} z^{D / 2-1} g_{\nu, D / 2-1}\left(\lambda z_{1}, \lambda z\right) e^{i k_{l} x^{l}},
$$

where we have introduced the function

$$
g_{\nu, \rho}(x, y)=Y_{\nu}(x) J_{\rho}(y)-J_{\nu}(x) Y_{\rho}(y) .
$$

From the boundary condition on $z=z_{2}$ we find that the allowed values of $\lambda$ are roots of the equation

$$
g_{\nu, \nu}\left(\lambda z_{1}, \lambda z_{2}\right)=0,
$$

with $\nu$ from (2.9). The positive solutions of this equation with respect to the first argument will be denoted by $\lambda_{\nu, n}=\lambda z_{1}, n=1,2, \ldots, \lambda_{\nu, n+1}>\lambda_{\nu, n}$. For the eigenvalues of the energy one obtains

$$
\omega_{\nu, n}=\sqrt{\lambda_{\nu, n}^{2} / z_{1}^{2}+k^{2}}
$$

Note that for $\nu= \pm 1 / 2$ we have

$$
g_{ \pm 1 / 2, \pm 1 / 2}\left(\lambda z_{1}, \lambda z\right)=-\frac{2 \sin \left[\lambda\left(z-z_{1}\right)\right]}{\pi \lambda \sqrt{z z_{1}}},
$$

and the corresponding eigenvalues are given by

$$
\lambda_{ \pm 1 / 2, n}=\frac{\pi n}{z_{2} / z_{1}-1} .
$$

These are the eigenvalues for the boundary condition (2.4) in the case $D=3$ and the eigenvalues for the boundary condition (2.5) in $D=3,5$. For the boundary condition (2.5), the modes (2.12) have been discussed in $[9,10]$ within the framework of $D=4$ Randall-Sundrum setup.

By using the condition (2.12), from (2.7), with the integration over $z$ in the range $z \in\left[z_{1}, z_{2}\right]$, for the coefficient $C$ one gets

$$
|C|^{2}=\frac{\lambda_{\nu, n} T_{\nu}\left(\eta, \lambda_{\nu, n}\right)}{8(2 \pi)^{D-4} \alpha^{D-3} z_{1}^{2} \omega_{\nu, n}}
$$

where

$$
\eta=z_{2} / z_{1}=e^{a / \alpha}
$$

and we have introduced the notation

$$
T_{\nu}(\eta, x)=x\left[\frac{J_{\nu}^{2}(x)}{J_{\nu}^{2}(x \eta)}-1\right]^{-1} .
$$

Note that $J_{\nu}\left(\lambda_{\nu, n}\right) / J_{\nu}\left(\lambda_{\nu, n} \eta\right)=Y_{\nu}\left(\lambda_{\nu, n}\right) / Y_{\nu}\left(\lambda_{\nu, n} \eta\right)$ and the expression (2.18) can also be written in terms of the Neumann function. With the normalization constant (2.16), the modes for the vector potential are completely specified.

Let us consider the mode functions in the limit $z_{1} \rightarrow 0$ (the left plate tends to the AdS boundary). For $\nu \geq 0$ one has $g_{\nu, \nu}\left(\lambda z_{1}, \lambda z_{2}\right) \approx Y_{\nu}\left(\lambda z_{1}\right) J_{\nu}\left(\lambda z_{2}\right)$ and the equation determining the eigenvalues of the radial quantum number $\lambda$ is reduced to $J_{\nu}\left(\lambda z_{2}\right)=0$. By taking into account (2.18), with the ratio of the Bessel functions replace by the ratio of the Neumann functions, we can see that

$$
C g_{\nu, D / 2-1}\left(\lambda z_{1}, \lambda z\right) \rightarrow C_{(\mathrm{I})} J_{D / 2-1}(\lambda z)
$$

with the normalization constant $\left|C_{(\mathrm{I})}\right|^{2}=2 /\left[(2 \pi)^{D-2} \alpha^{D-3} \times\right.$ $\left.\omega z_{2}^{2} J_{\nu}^{\prime 2}\left(\lambda z_{2}\right)\right]$. For the boundary condition (2.4) the mode functions obtained in this way coincide with those considered in $[20,22]$ for the geometry of a single plate. For the boundary condition (2.5) and for $D=3$ one has $\nu=-1 / 2$ the equation for the eigenvalues of $\lambda$ is reduced to $Y_{\nu}\left(\lambda z_{2}\right)=0$ which is equivalent to $\sin \left(\lambda z_{2}\right)=0$. For the radial part of the mode functions we get

$$
C z^{D / 2-1} g_{\nu, D / 2-1}\left(\lambda z_{1}, \lambda z\right) \rightarrow \frac{\cos (\lambda z)}{\sqrt{\pi z_{2} \omega}}
$$

Notice that for $D=3$ and in the case of the boundary condition (2.4) the eigenvalue equation is the same, $\sin \left(\lambda z_{2}\right)=0$, and the mode functions are obtained from (2.20) by the replacement $\cos (\lambda z) \rightarrow \sin (\lambda z)$.

In the geometry of a single plate at $z=z_{2}$ and for the region $0 \leq z \leq z_{2}$ the mode functions have the form (2.6). The integral over $z$ in the normalization condition (2.7) is reduced to $\int_{0}^{z_{2}} d z z Z_{D / 2-1}\left(\lambda^{\prime} z\right) Z_{D / 2-1}(\lambda z)$, where $Z_{\mu}(x)=$ $c_{1} J_{\mu}(x)+c_{2} Y_{\mu}(x)$. For $c_{2} \neq 0$, near the lower limit the integrand behaves as $z^{3-D}$. From here it follows that for $D \geq 4$ and for normalizable modes one should take $c_{2}=0$. Imposing the boundary condition (2.4) or (2.5) on $z=z_{2}$ we see that the eigenvalues for $\lambda$ are the roots of $J_{\nu}\left(\lambda z_{2}\right)=0$ with $\nu$ given by (2.9). These are the modes we have obtained by the limiting transition $z_{1} \rightarrow 0$. For $D=3$ the mode functions with $c_{2} \neq 0$ are normalizable and in order to uniquely specify them an additional boundary condition is required on the AdS boundary. As a result of the limiting transition $z_{1} \rightarrow 0$, depending on the conditions 
imposed at $z=z_{1}$, we have obtained two special types of boundary conditions.

\section{TWO-POINT FUNCTIONS FOR THE VECTOR POTENTIAL}

The two-point functions of a free-field theory are important characteristics of quantum fields describing the correlations of fluctuations at different spacetime points. Having these functions one can evaluate the VEVs for various physical quantities like the field squared, the energy-momentum tensor and the current density. The free-field two-point functions are the building blocks in the perturbative expansion of correlation functions in interacting field theories. First we consider the two-point function for the vector potential in the region between the plates (region II). With the mode functions (2.10) and the eigenvalues of $\lambda$ determined from (2.12), the two-point function (the positive-frequency Wightman function)

$$
\left\langle 0\left|A_{\mu}(x) A_{\rho}\left(x^{\prime}\right)\right| 0\right\rangle \equiv\left\langle A_{\mu} A_{\rho}^{\prime}\right\rangle
$$

is evaluated by using the mode-sum formula

$$
\left\langle A_{\mu} A_{\rho}^{\prime}\right\rangle=\int d \mathbf{k} \sum_{n=1}^{\infty} \sum_{\sigma=1}^{D-1} A_{(\beta) \mu}(x) A_{(\beta) \rho}\left(x^{\prime}\right),
$$

where $|0\rangle$ stands for the vacuum state and $\int d \mathbf{k}=$ $\int_{-\infty}^{+\infty} d k_{1} \cdots \int_{-\infty}^{+\infty} d k_{D-1}$. The summation over the polarizations is done by using the formula

$$
\sum_{\sigma=1}^{D-1} \epsilon_{(\sigma) \mu} \epsilon_{(\sigma) \rho}=\frac{k_{\mu} k_{\rho}}{\lambda^{2}}-\eta_{\mu \rho}
$$

and for the nonzero components one finds

$$
\begin{aligned}
\left\langle A_{\mu} A_{\rho}^{\prime}\right\rangle= & \frac{\left(z z^{\prime}\right)^{D / 2-1}}{8(2 \pi)^{D-4} \alpha^{D-3}} \int d \mathbf{k} \sum_{n=1}^{\infty} \frac{\lambda_{\nu, n} T_{\nu}\left(\eta, \lambda_{\nu, n}\right)}{\sqrt{\lambda_{\nu, n}^{2} / z_{1}^{2}+k^{2}}} e^{i k_{l} \Delta x^{l}} \\
& \times g_{\nu, D / 2-1}\left(\lambda_{\nu, n}, \lambda_{\nu, n} z / z_{1}\right) \\
& \times g_{\nu, D / 2-1}\left(\lambda_{\nu, n}, \lambda_{\nu, n} z^{\prime} / z_{1}\right)\left(\frac{k_{\mu} k_{\rho}}{\lambda_{\nu, n}^{2}}-\frac{\eta_{\mu \rho}}{z_{1}^{2}}\right)
\end{aligned}
$$

where $k_{0}=\omega_{\nu, n}, \Delta x^{l}=x^{l}-x^{\prime l}, \mu, \rho, l=0,1, \ldots, D-1$.

In (3.4) the eigenvalues $\lambda_{\nu, n}$ for general spatial dimension are given implicitly and this representation is not convenient for the evaluation of the VEVs in the coincidence limit of the arguments. A more adapted representation is obtained by making use of a variant of the generalized Abel-Plana formula [26,27]

$$
\begin{aligned}
\sum_{n=1}^{\infty} & f\left(\lambda_{\nu, n}\right) T_{\nu}\left(\eta, \lambda_{\nu, n}\right) \\
= & \frac{2}{\pi^{2}} \int_{0}^{\infty} \frac{f(u) d u}{J_{\nu}^{2}(u)+Y_{\nu}^{2}(u)} \\
& -\frac{1}{2 \pi} \int_{0}^{\infty} d u \Omega_{\nu}^{(1)}(u, \eta u)[f(i u)+f(-i u)],
\end{aligned}
$$

with the notations

$$
\Omega_{\nu}^{(1)}(x, y)=\frac{K_{\nu}(y)}{K_{\nu}(x) G_{\nu, \nu}(x, y)}
$$

and

$$
G_{\nu, \rho}(x, y)=K_{\nu}(x) I_{\rho}(y)-(-1)^{\nu-\rho} I_{\nu}(x) K_{\rho}(y) .
$$

Here, $I_{\nu}(x)$ and $K_{\nu}(x)$ are the modified Bessel functions. In the case of the function $f(u)$ corresponding to (3.4), the conditions of the validity for (3.5) are satisfied if $z+z^{\prime}+|\Delta t|<2 z_{2}$. In the coincidence limit and for the region between the plates this condition is obeyed for points away from the plate at $z=z_{2}$.

For the series in (3.4) the function $f(u)$ is given by the expression

$$
\begin{aligned}
f(u)= & \frac{u e^{-i \sqrt{u^{2} / z_{1}^{2}+k^{2}} \Delta t}}{\sqrt{u^{2} / z_{1}^{2}+k^{2}}} g_{\nu, D / 2-1}\left(u, u z / z_{1}\right) \\
& \times g_{\nu, D / 2-1}\left(u, u z^{\prime} / z_{1}\right)\left(\frac{k_{\mu} k_{\rho}}{u^{2}}-\frac{\eta_{\mu \rho}}{z_{1}^{2}}\right) .
\end{aligned}
$$

By applying (3.5), the two-point function is presented in the decomposed form

$$
\begin{aligned}
\left\langle A_{\mu} A_{\rho}^{\prime}\right\rangle= & \left\langle A_{\mu} A_{\rho}^{\prime}\right\rangle_{1}+\frac{4 \delta_{\nu}\left(z z^{\prime}\right)^{D / 2-1}}{(2 \pi)^{D-1} \alpha^{D-3}} \int d \mathbf{k} \int_{k}^{\infty} d u \\
& \times\left(\eta_{\mu \rho} u^{2}+\partial_{\mu} \partial_{\rho}^{\prime}\right) e^{i k_{l} \Delta x^{\prime}} \frac{\Omega_{\nu}^{(1)}\left(u z_{1}, u z_{2}\right)}{u \sqrt{u^{2}-k^{2}}} \\
& \times G_{\nu, D / 2-1}\left(u z_{1}, u z\right) G_{\nu, D / 2-1}\left(u z_{1}, u z^{\prime}\right) \\
& \times \cosh \left(\Delta t \sqrt{u^{2}-k^{2}}\right)
\end{aligned}
$$

for $\mu, \rho=0,1, \ldots, D-1$ and $l=1, \ldots, D-1$ for the summation in $k_{l} \Delta x^{l}$. Here we have defined

$$
\delta_{\nu}= \begin{cases}1, & \text { for } \nu=D / 2-1 \\ -1, & \text { for } \nu=D / 2-2\end{cases}
$$

The first term in the right-hand side of (3.9) is given by the expression 


$$
\begin{aligned}
\left\langle A_{\mu} A_{\rho}^{\prime}\right\rangle_{1}= & \frac{\left(z z^{\prime}\right)^{D / 2-1}}{(2 \pi)^{D-2} \alpha^{D-3}} \int d \mathbf{k} \int_{0}^{\infty} d \lambda \frac{\lambda}{\omega}\left(\frac{k_{\mu} k_{\rho}}{\lambda^{2}}-\eta_{\mu \rho}\right) \\
& \times \frac{g_{\nu, D / 2-1}\left(\lambda z_{1}, \lambda z\right) g_{\nu, D / 2-1}\left(\lambda z_{1}, \lambda z^{\prime}\right)}{J_{\nu}^{2}\left(\lambda z_{1}\right)+Y_{\nu}^{2}\left(\lambda z_{1}\right)} e^{i k_{l} \Delta x^{l}},
\end{aligned}
$$

where $\omega=\sqrt{\lambda^{2}+k^{2}}$ and $l=0,1, \ldots, D-1$.

In the limit $z_{2} \rightarrow \infty$ the last term in (3.9) tends to zero and the part (3.11) is interpreted as the two-point function in the region $z>z_{1}$ for the geometry of a single plate at $z=z_{1}$. This can also be seen directly by evaluating the corresponding mode sum. The mode functions are still given by (2.10) where now the eigenvalues of $\lambda$ are continuous. The normalization constant is obtained from (2.7) with the $z$ integral over the region $z_{1} \leq z<\infty$ and is given by

$$
\left|C_{(\mathrm{III})}\right|^{2}=\frac{(2 \pi)^{2-D} \alpha^{3-D} \lambda}{\omega\left[J_{\nu}^{2}\left(\lambda z_{1}\right)+Y_{\nu}^{2}\left(\lambda z_{1}\right)\right]} .
$$

The mode sum with the functions (2.10) and the normalization coefficient (3.12) leads to the representation (3.11). The boundary-induced contribution in (3.11) can be separated in a way similar to that used in [21] for the special case of the boundary condition (2.4). The corresponding expression reads

$$
\begin{aligned}
\left\langle A_{\mu} A_{\rho}^{\prime}\right\rangle_{1}= & \left\langle A_{\mu} A_{\rho}^{\prime}\right\rangle_{0}+\frac{4 \delta_{\nu}\left(z z^{\prime}\right)^{D / 2-1}}{(2 \pi)^{D-1} \alpha^{D-3}} \\
& \times \int d \mathbf{k} \int_{k}^{\infty} d u\left(\eta_{\mu \rho} x^{2}+\partial_{\mu} \partial_{\rho}^{\prime}\right) e^{i k_{l} \Delta x^{l}} \\
& \times \frac{\cosh \left(\Delta t \sqrt{u^{2}-k^{2}}\right)}{u \sqrt{u^{2}-k^{2}}} \frac{I_{\nu}\left(u z_{1}\right)}{K_{\nu}\left(u z_{1}\right)} \\
& \times K_{D / 2-1}(u z) K_{D / 2-1}\left(u z^{\prime}\right)
\end{aligned}
$$

where $l=0,1, \ldots, D-1$ and $\left\langle A_{\mu} A_{\rho}^{\prime}\right\rangle_{0}$ is the two-point function in $\mathrm{AdS}$ spacetime when the plates are absent.

An alternative representation for the two-point function of the vector potential in the region between the plates is obtained by using the relation

$$
\begin{aligned}
& \frac{I_{\nu}\left(u z_{1}\right)}{K_{\nu}\left(u z_{1}\right)} K_{D / 2-1}(u z) K_{D / 2-1}\left(u z^{\prime}\right) \\
& \quad-\frac{K_{\nu}\left(u z_{2}\right)}{I_{\nu}\left(u z_{2}\right)} I_{D / 2-1}(u z) I_{D / 2-1}\left(u z^{\prime}\right) \\
& =\sum_{j=1,2}(-1)^{j} \Omega_{\nu}^{(j)}\left(u z_{1}, u z_{2}\right) G_{\nu, D / 2-1}\left(u z_{j}, u z\right) \\
& \quad \times G_{\nu, D / 2-1}\left(u z_{j}, u z^{\prime}\right)
\end{aligned}
$$

where

$$
\Omega_{\nu}^{(2)}(x, y)=\frac{I_{\nu}(x)}{I_{\nu}(y) G_{\nu, \nu}(x, y)} .
$$

This leads to the expression

$$
\begin{aligned}
\left\langle A_{\mu} A_{\rho}^{\prime}\right\rangle= & \left\langle A_{\mu} A_{\rho}^{\prime}\right\rangle_{2}+\frac{4 \delta_{\nu}\left(z z^{\prime}\right)^{D / 2-1}}{(2 \pi)^{D-1} \alpha^{D-3}} \int d \mathbf{k} \int_{k}^{\infty} d u \\
& \times\left(\eta_{\mu \rho} u^{2}+\partial_{\mu} \partial_{\rho}^{\prime}\right) e^{i k_{l} \Delta x^{l}} \frac{\Omega_{\nu}^{(2)}\left(u z_{1}, u z_{2}\right)}{u \sqrt{u^{2}-k^{2}}} \\
& \times G_{\nu, D / 2-1}\left(u z_{2}, u z\right) G_{\nu, D / 2-1}\left(u z_{2}, u z^{\prime}\right) \\
& \times \cosh \left(\Delta t \sqrt{u^{2}-k^{2}}\right)
\end{aligned}
$$

where

$$
\begin{aligned}
\left\langle A_{\mu} A_{\rho}^{\prime}\right\rangle_{2}= & \left\langle A_{\mu} A_{\rho}^{\prime}\right\rangle_{0}+\frac{4 \delta_{\nu}\left(z z^{\prime}\right)^{D / 2-1}}{(2 \pi)^{D-1} \alpha^{D-3}} \\
& \times \int d \mathbf{k} \int_{k}^{\infty} d u\left(\eta_{\mu \rho} u^{2}+\partial_{\mu} \partial_{\rho}^{\prime}\right) e^{i k_{l} \Delta x^{l}} \\
& \times \frac{\cosh \left(\Delta t \sqrt{u^{2}-k^{2}}\right)}{u \sqrt{u^{2}-k^{2}}} \frac{K_{\nu}\left(u z_{2}\right)}{I_{\nu}\left(u z_{2}\right)} \\
& \times I_{D / 2-1}(u z) I_{D / 2-1}\left(u z^{\prime}\right)
\end{aligned}
$$

is the two-point function in the region $0 \leq z \leq z_{2}$ for the geometry of a single plate at $z=z_{2}$.

In region I the two-point function is given by (3.17) with the replacement $z_{2} \rightarrow z_{1}$. For region III, $z_{2} \leq z<\infty$, the two-point function is obtained from (3.13) replacing $z_{2} \rightarrow z_{1}$. The corresponding result could also be obtained directly considering the field dynamics in that region. The mode functions are given by (2.10) with the replacement (2.19) and $z_{2} \rightarrow z_{1}$ and the eigenvalues for $\lambda$ are the zeros of the function $J_{\nu}\left(\lambda z_{1}\right)$. The series over these zeros in the mode sum for the two-point function $\left\langle A_{\mu} A_{\rho}^{\prime}\right\rangle_{1}$ is summed by using the respective formula from $[26,27]$. That allows to separate the plate-induced contribution explicitly.

\section{CORRELATORS FOR THE FIELD TENSOR}

The VEVs of physical observables bilinear in the fields, such as the fields squared and energy-momentum tensor, are obtained from the two-point function for the field strength tensor

$$
\left\langle 0\left|F_{\mu \sigma}(x) F_{\kappa \rho}\left(x^{\prime}\right)\right| 0\right\rangle=\left\langle F_{\mu \sigma} F_{\kappa \rho}^{\prime}\right\rangle .
$$

Given the plate-induced contributions to the two-point function for the vector potential we can find the corresponding contributions to the correlator (4.1) by differentiations. First of all for the components with $\mu, \sigma, \kappa, \rho=$ $1, \ldots, D-1$ we get 


$$
\begin{aligned}
\left\langle F_{\mu \sigma} F_{\kappa \rho}^{\prime}\right\rangle= & \left\langle F_{\mu \sigma} F_{\kappa \rho}^{\prime}\right\rangle_{j}+\frac{8 \delta_{\nu}\left(z z^{\prime}\right)^{D / 2-1}}{(2 \pi)^{D-1}} \alpha^{D-3} \int d \mathbf{k} e^{i k_{l} \Delta x^{l}} \int_{k}^{\infty} d u u \\
& \times \frac{\cosh \left(\Delta t \sqrt{u^{2}-k^{2}}\right)}{\sqrt{u^{2}-k^{2}}} F_{\nu}^{(j)}\left(u z, u z^{\prime}\right) \\
& \times\left(\eta_{[\sigma \rho} k_{\mu]} k_{\kappa}+\eta_{[\mu \kappa} k_{\sigma]} k_{\rho}\right)
\end{aligned}
$$

$$
\begin{aligned}
\left\langle F_{\mu D} F_{\kappa D}^{\prime}\right\rangle= & \left\langle F_{\mu D} F_{\kappa D}^{\prime}\right\rangle_{j} \\
& +\frac{4 \delta_{\nu} \alpha^{3-D}}{(2 \pi)^{D-1}} \int d \mathbf{k} e^{i k_{l} \Delta x^{l}} \int_{k}^{\infty} d u u\left(\frac{k_{\mu} k_{\kappa}}{u^{2}}+\eta_{\mu \kappa}\right) \\
& \times \frac{\cosh \left(\Delta t \sqrt{u^{2}-k^{2}}\right)}{\sqrt{u^{2}-k^{2}}} \\
& \times \partial_{z} \partial_{z^{\prime}}\left(\left(z z^{\prime}\right)^{D / 2-1} F_{\nu}^{(j)}\left(u z, u z^{\prime}\right)\right]
\end{aligned}
$$

where $j=1,2, l=1, \ldots, D-1$, the square brackets in the index expression mean antisymmetrization with respect to the corresponding indices [indices $\sigma$ and $\mu$ in (4.2)] and

$$
\begin{aligned}
F_{\nu}^{(j)}\left(x z, x z^{\prime}\right)= & \Omega_{\nu}^{(j)}\left(x z_{1}, x z_{2}\right) G_{\nu, D / 2-1}\left(x z_{j}, x z\right) \\
& \times G_{\nu, D / 2-1}\left(x z_{j}, x z^{\prime}\right)
\end{aligned}
$$

Here, $j=1$ and $j=2$ provide two equivalent representations. These representations are also valid for $\mu=\kappa=0$ if $k_{0}$ is understood as $k_{0}=\sqrt{k^{2}-u^{2}}$. For the components with only one of the indices being 0 and $D$ we have

$$
\begin{aligned}
\left\langle F_{0 \mu} F_{\rho \kappa}^{\prime}\right\rangle= & \left\langle F_{0 \mu} F_{\rho \kappa}^{\prime}\right\rangle_{j}-\frac{8 i \delta_{\nu}\left(z z^{\prime}\right)^{D / 2-1}}{(2 \pi)^{D-1} \alpha^{D-3}} \int d \mathbf{k} e^{i k_{l} \Delta x^{l}} \\
& \times \int_{k}^{\infty} d u u \sinh \left(\Delta t \sqrt{u^{2}-k^{2}}\right) F_{\nu}^{(j)}\left(u z, u z^{\prime}\right) \delta_{\mu[\kappa} k_{\rho]},
\end{aligned}
$$

$$
\begin{aligned}
\left\langle F_{D \mu} F_{\rho \kappa}^{\prime}\right\rangle= & \left\langle F_{D \mu} F_{\rho \kappa}^{\prime}\right\rangle_{j}-\frac{8 i \delta_{\nu} z^{\prime D / 2-1}}{(2 \pi)^{D-1} \alpha^{D-3}} \int d \mathbf{k} e^{i k_{l} \Delta x^{l}} \\
& \times \int_{k}^{\infty} d u u \frac{\cosh \left(\sqrt{u^{2}-k^{2}} \Delta t\right)}{\sqrt{u^{2}-k^{2}}} \\
& \times \partial_{z}\left[z^{D / 2-1} F_{\nu}^{(j)}\left(u z, u z^{\prime}\right)\right] \delta_{\mu[\kappa} k_{\rho]} .
\end{aligned}
$$

The remaining components are found by using the relation

$$
\left\langle F_{\mu \sigma} F_{\kappa \rho}^{\prime}\right\rangle=\left\langle F_{\kappa \rho}^{\prime} F_{\mu \sigma}\right\rangle^{*},
$$

valid for all values of the indices. The parts $\left\langle F_{\mu \sigma} F_{\kappa \rho}^{\prime}\right\rangle_{j}$ in (4.2)-(4.6) present the two-point functions in the geometry for a single plate at $z=z_{j}$. They are decomposed as

$$
\left\langle F_{\mu \sigma} F_{\kappa \rho}^{\prime}\right\rangle_{j}=\left\langle F_{\mu \sigma} F_{\kappa \rho}^{\prime}\right\rangle_{0}+\left\langle F_{\mu \sigma} F_{\kappa \rho}^{\prime}\right\rangle_{j}^{(\mathrm{b})},
$$

where $\left\langle F_{\mu \sigma} F_{\kappa \rho}^{\prime}\right\rangle_{0}$ is the corresponding function in the geometry without plates and the part $\left\langle F_{\mu \sigma} F_{\kappa \rho}^{\prime}\right\rangle_{j}^{(b)}$ is induced by a single plate at $z=z_{j}$. The expressions for $\left\langle F_{\mu \sigma} F_{\kappa \rho}^{\prime}\right\rangle_{j}^{(\mathrm{b})}$ are obtained from the last terms in (4.2), (4.3), (4.5) and (4.6) by the replacement

$$
F_{\nu}^{(j)}\left(x z, x z^{\prime}\right) \rightarrow \frac{K_{\nu}\left(u z_{j}\right)}{I_{\nu}\left(u z_{j}\right)} I_{D / 2-1}(u z) I_{D / 2-1}\left(u z^{\prime}\right)
$$

for the region $z, z^{\prime}<z_{j}$ and by the replacement

$$
F_{\nu}^{(j)}\left(x z, x z^{\prime}\right) \rightarrow \frac{I_{\nu}\left(u z_{j}\right)}{K_{\nu}\left(u z_{j}\right)} K_{D / 2-1}(u z) K_{D / 2-1}\left(u z^{\prime}\right)
$$

in the region $z, z^{\prime}>z_{j}$. The last terms in (4.2), (4.3), (4.5) and (4.6) are induced by the plate $z=z_{j^{\prime}}, j^{\prime}=1,2, j^{\prime} \neq j$, when one adds it to the geometry with a single plate at $z=z_{j}$.

In the evaluation of the local VEVs we need the twopoint functions in the coincidence limit $x^{\prime} \rightarrow x$. For $\mu, \sigma, \kappa, \rho=0,1, \ldots, D-1$ and in the coincidence limit, for the nonzero components one finds

$$
\begin{aligned}
\left\langle F_{\mu \sigma} F_{\kappa \rho}\right\rangle= & \left\langle F_{\mu \sigma} F_{\kappa \rho}\right\rangle_{j}-\frac{2 \delta_{\nu}\left(\eta_{\mu \kappa} \eta_{\sigma \rho}-\eta_{\mu \rho} \eta_{\sigma \kappa}\right) z^{D-2}}{(4 \pi)^{D / 2-1} \Gamma(D / 2+1) \alpha^{D-3}} \\
& \times \int_{0}^{\infty} d u u^{D+1} \Omega_{\nu}^{(j)}\left(u z_{1}, u z_{2}\right) G_{\nu, D / 2-1}^{2}\left(u z_{j}, u z\right), \\
\left\langle F_{\mu D} F_{\kappa D}\right\rangle= & \left\langle F_{\mu D} F_{\kappa D}\right\rangle_{j}+\frac{\delta_{\nu} \eta_{\mu \kappa}(D-1) \alpha^{3-D} z^{D-2}}{(4 \pi)^{D / 2-1} \Gamma(D / 2+1)} \\
& \times \int_{0}^{\infty} d u u^{D+1} \Omega_{\nu}^{(j)}\left(u z_{1}, u z_{2}\right) G_{\nu, D / 2-2}^{2}\left(u z_{j}, u z\right),
\end{aligned}
$$

where the relation

$$
\partial_{z}\left[z^{D / 2-1} G_{\nu, D / 2-1}\left(u z_{j}, u z\right)\right]=u z^{D / 2-1} G_{\nu, D / 2-2}\left(u z_{j}, u z\right)
$$

has been used. The boundary-induced contributions in the $\operatorname{VEVs}\left\langle F_{\mu \sigma} F_{\kappa \rho}\right\rangle_{j}$ for the geometry of a single plate at $z=z_{j}$ are obtained from the last terms in (4.11) and (4.12) by the replacements (4.9) and (4.10) with $z^{\prime}=z$. Note that for points away from the boundaries the divergences in the coincidence limit are contained in the parts $\left\langle F_{\mu \sigma} F_{\kappa \rho}\right\rangle_{0}$ only. In the representations given above the contribution $\left\langle F_{\mu \sigma} F_{\kappa \rho}\right\rangle_{0}$ is explicitly extracted and consequently the renormalization of the local observables at points outside of plates is reduced to the renormalization in the geometry without plates. In the following sections the expressions 
(4.11) and (4.12) are used for the evaluation of the VEVs of fields squared and of the energy-momentum tensor.

\section{VEVS OF THE ELECTRIC AND MAGNETIC FIELDS SQUARED AND PHOTON CONDENSATE}

We start the investigation of the local VEVs from the electric field squared. Having the two-point functions of the field tensor in the coincidence limit, this VEV is evaluated by using the relation

$$
\left\langle E^{2}\right\rangle=-g^{00} g^{\mu \rho}\left\langle F_{0 \mu} F_{0 \rho}\right\rangle .
$$

From (4.11) and (4.12), in the region $z_{1}<z<z_{2}$ one gets

$$
\begin{aligned}
\left\langle E^{2}\right\rangle= & \left\langle E^{2}\right\rangle_{j}+\frac{\delta_{\nu}(D-1) \alpha^{-1-D} z^{D+2}}{(4 \pi)^{D / 2-1} \Gamma(D / 2+1)} \\
& \times \int_{0}^{\infty} d x x^{D+1} \Omega_{\nu}^{(j)}\left(x z_{1}, x z_{2}\right) \\
& \times\left[2 G_{\nu, D / 2-1}^{2}\left(x z_{j}, x z\right)+G_{\nu, D / 2-2}^{2}\left(x z_{j}, x z\right)\right] .
\end{aligned}
$$

Here, $\left\langle E^{2}\right\rangle_{j}$ is the VEV of the electric field squared for the geometry of a single plate at $z=z_{j}$ in the region $z>z_{1}$ for $j=1$ and $z<z_{2}$ for $j=2$. For $j=1$ the VEV is given by the expressions

$$
\begin{aligned}
\left\langle E^{2}\right\rangle_{1}= & \left\langle E^{2}\right\rangle_{0}+\frac{\delta_{\nu}(D-1) \alpha^{-1-D} z^{D+2}}{(4 \pi)^{D / 2-1} \Gamma(D / 2+1)} \\
& \times \int_{0}^{\infty} d x x^{D+1} \frac{I_{\nu}\left(x z_{1}\right)}{K_{\nu}\left(x z_{1}\right)}\left[2 K_{D / 2-1}^{2}(x z)+K_{D / 2-2}^{2}(x z)\right],
\end{aligned}
$$

where $\left\langle E^{2}\right\rangle_{0}$ is the VEV in the absence of the plates. For $j=2$ the expression $\left\langle E^{2}\right\rangle_{2}$ in (5.2) is obtained from (5.3) by the replacements $z_{1} \rightarrow z_{2}$ and $I \rightleftarrows K$. In the region $z<z_{1}$ the VEV of the electric field squared is given by (5.3) with the replacements $I \rightleftarrows K$ [in the special case of boundary condition (2.4) the sign difference with the result in [20] is related to the definition of $\left\langle E^{2}\right\rangle$ in (5.1) without the minus sign in the right-hand side]. The VEV for the region $z>z_{2}$ is obtained from (5.3) by the replacement $z_{1} \rightarrow z_{2}$. The second term in the right-hand side of (5.2) is interpreted as the contribution induced by the second plate when we add it to the geometry of a single plate at $z=z_{j}$. Note that $G_{\mu, \mu}(x, y)>0$ for $y>x>0$ and, hence, $\Omega_{\nu}^{(j)}(x, y)>0$. From here we conclude that both the singleplate-induced [the last term in (5.3)] and the second-plateinduced [the last term in (5.2)] contributions to the VEV of the electric field squared are positive (negative) for the boundary condition (2.4) [(2.5)].

The VEV (5.2) diverges on the plates. The divergences come from the single plate contributions $\left\langle E^{2}\right\rangle_{j}$ and the second plate contribution in (5.2) is finite on the plate $z=z_{j}$. In order to find the leading term in the asymptotic expansion over the distance from the plate we note that for points near the plate the dominant contribution to the integral in (5.3) (and in the analog integral for $\left\langle E^{2}\right\rangle_{2}$ ) comes from large values of $x$ and we can use the corresponding asymptotics for the modified Bessel functions. For $\left|z / z_{j}-1\right| \ll 1$, to the leading order this gives

$$
\left\langle E^{2}\right\rangle \approx\left\langle E^{2}\right\rangle_{j} \approx \delta_{\nu} \frac{3(D-1) \Gamma((D+1) / 2) \alpha^{-1-D}}{2(4 \pi)^{(D-1) / 2}\left|1-z_{j} / z\right|^{D+1}} .
$$

Note that in the asymptotic region under consideration $\left|1-z_{j} / z\right| \approx\left|y-y_{j}\right| / \alpha$ and in terms of the coordinate $y$ the leading term does not depend on the curvature radius. To the leading order the VEV coincides with that for plates in the Minkowski spacetime (see below). This feature was expected by taking into account that near the plates the dominant contribution to the VEVs comes from the fluctuations with small wavelengths (compared with the curvature radius) and the influence of the gravitational field on those modes is weak.

In a similar way we can find the $\operatorname{VEV}\left\langle F_{\mu \sigma} F^{\mu \sigma}\right\rangle$. It is the analog of the gluon condensate in quantum chromodynamics and is known as photon condensate (see, for instance, [28]). From (4.11) and (4.12) we find

$$
\begin{aligned}
\left\langle F_{\mu \sigma} F^{\mu \sigma}\right\rangle= & \left\langle F_{\mu \sigma} F^{\mu \sigma}\right\rangle_{j}-\frac{4 \delta_{\nu}(D-1) z^{D+2}}{(4 \pi)^{D / 2-1} \Gamma(D / 2) \alpha^{D+1}} \\
& \times \int_{0}^{\infty} d x x^{D+1} \Omega_{\nu}^{(j)}\left(x z_{1}, x z_{2}\right) \\
& \times\left[G_{\nu, D / 2-1}^{2}\left(u z_{j}, u z\right)+G_{\nu, D / 2-2}^{2}\left(u z_{j}, u z\right)\right],
\end{aligned}
$$

where

$$
\begin{aligned}
\left\langle F_{\mu \sigma} F^{\mu \sigma}\right\rangle_{1}= & \left\langle F_{\mu \sigma} F^{\mu \sigma}\right\rangle_{0}-\frac{4 \delta_{\nu}(D-1) z^{D+2}}{(4 \pi)^{D / 2-1} \Gamma(D / 2) \alpha^{D+1}} \\
& \times \int_{0}^{\infty} d x x^{D+1} \frac{I_{\nu}\left(x z_{1}\right)}{K_{\nu}\left(x z_{1}\right)} \\
& \times\left[K_{D / 2-1}^{2}(x z)+K_{D / 2-2}^{2}(x z)\right]
\end{aligned}
$$

is the corresponding VEV in the region $z>z_{1}$ for the geometry of a single plate at $z=z_{1}$. For $j=2$, the term $\left\langle F_{\beta \sigma} F^{\beta \sigma}\right\rangle_{2}$ is the VEV in the geometry of a single plate at $z=z_{2}$ for the region $z<z_{2}$. The boundary-induced contribution to the photon condensate is negative for the boundary condition (2.4) and positive for the condition (2.5). Near the plates one has the asymptotic $\left\langle F_{\mu \sigma} F^{\mu \sigma}\right\rangle \approx$ $-2\left\langle E^{2}\right\rangle / 3$ with $\left\langle E^{2}\right\rangle$ given by (5.4).

Having the VEVs of the electric field squared and the photon condensate we can find the VEV of the magnetic field squared by using the relation $\left\langle B^{2}\right\rangle=\left\langle E^{2}\right\rangle+$ $\left\langle F_{\mu \sigma} F^{\mu \sigma}\right\rangle / 2$. Note that in dimensions $D>3$ the magnetic 
field is not a spatial vector and the corresponding VEV is given by

$$
\left\langle B^{2}\right\rangle=\frac{1}{2} g^{l m} g^{n p}\left\langle F_{l n} F_{m p}\right\rangle,
$$

with $l, m, n, p=1,2, \ldots, D$. From (5.2) and (5.5) one gets

$$
\begin{aligned}
\left\langle B^{2}\right\rangle= & \left\langle B^{2}\right\rangle_{j}-\frac{\delta_{\nu}(D-1) \alpha^{-1-D} z^{D+2}}{(4 \pi)^{D / 2-1} \Gamma(D / 2+1)} \\
& \times \int_{0}^{\infty} d x x^{D+1} \Omega_{\nu}^{(j)}\left(x z_{1}, x z_{2}\right) \\
& \times\left[(D-2) G_{\nu, D / 2-1}^{2}\left(x z_{j}, x z\right)\right. \\
& \left.+(D-1) G_{\nu, D / 2-2}^{2}\left(x z_{j}, x z\right)\right] .
\end{aligned}
$$

Here the part corresponding to a single plate geometry is defined as

$$
\begin{aligned}
\left\langle B^{2}\right\rangle_{1}= & \left\langle B^{2}\right\rangle_{0}-\frac{\delta_{\nu}(D-1) \alpha^{-1-D} z^{D+2}}{(4 \pi)^{D / 2-1} \Gamma(D / 2+1)} \int_{0}^{\infty} d x x^{D+1} \frac{I_{\nu}\left(x z_{1}\right)}{K_{\nu}\left(x z_{1}\right)} \\
& \times\left[(D-2) K_{D / 2-1}^{2}(x z)+(D-1) K_{D / 2-2}^{2}(x z)\right]
\end{aligned}
$$

for $j=1$ and the expression for $\left\langle B^{2}\right\rangle_{2}$ is obtained from (5.9) by the replacements $I \rightleftarrows K$ and $z_{1} \rightarrow z_{2}$ in the boundary-induced contribution. In a way similar to that for the electric field squared we can see that the singleplate-induced [the last term in (5.9)] and the second-plateinduced [the last term in (5.8)] contributions to the VEV of the magnetic field squared are negative (positive) for the boundary condition (2.4) [(2.5)]. Near the plates the VEV of the magnetic field squared behaves like $\left\langle B^{2}\right\rangle \approx$ $(1-2 D / 3)\left\langle E^{2}\right\rangle$, where the asymptotic for $\left\langle E^{2}\right\rangle$ near the plate $z=z_{j}$ is given by (5.4).

For $D=3$, by using the expressions for the functions $I_{ \pm 1 / 2}(x)$ and $K_{ \pm 1 / 2}(x)$, we can see that

$$
\begin{aligned}
G_{ \pm 1 / 2, \pm 1 / 2}\left(x z_{j}, x z\right) & =\frac{\sinh \left[x\left(z-z_{j}\right)\right]}{x \sqrt{z z_{j}}}, \\
G_{ \pm 1 / 2, \mp 1 / 2}\left(x z_{j}, x z\right) & =\frac{\cosh \left[x\left(z-z_{j}\right)\right]}{x \sqrt{z z_{j}}} .
\end{aligned}
$$

With these expressions, from (5.2), (5.3), (5.8) and (5.9) one finds

$$
\begin{aligned}
\left\langle F^{2}\right\rangle= & \left\langle F^{2}\right\rangle_{0}-\frac{3 \delta_{(F)}}{4 \pi \alpha^{4}}-\frac{z^{4} \delta_{(F)}}{4 \pi \alpha^{4}}\left[\frac{\delta_{(F)} \pi^{4} / 45}{\left(z_{2}-z_{1}\right)^{4}} \mp \frac{3}{\left(z-z_{1}\right)^{4}}\right. \\
& \left.\mp \int_{0}^{\infty} d x \frac{x^{3} \cosh \left[x\left(z-z_{1}\right)\right]}{e^{x\left(z_{2}-z_{1}\right)}-1}\right]
\end{aligned}
$$

where $F=E, B$ for the electric and magnetic fields, respectively, and

$$
\delta_{(E)}=-\delta_{(B)}=1 .
$$

The upper and lower signs in (5.11) correspond to the boundary conditions (2.4) and (2.5), respectively. An alternative expression is obtained by using the expansion $1 /\left(e^{u}-1\right)=\sum_{n=1}^{\infty} e^{-n u}$ in the integral term:

$$
\begin{aligned}
\left\langle F^{2}\right\rangle= & \left\langle F^{2}\right\rangle_{0}-\frac{3 \delta_{(F)}}{4 \pi \alpha^{4}}-\frac{\alpha^{-4} z^{4}}{4 \pi\left(z_{2}-z_{1}\right)^{4}} \\
& \times\left[\frac{\pi^{4}}{45} \mp 3 \delta_{(F)} \sum_{n=-\infty}^{\infty}\left(n-\frac{z-z_{1}}{z_{2}-z_{1}}\right)^{-4}\right],
\end{aligned}
$$

again, with the upper and lower signs corresponding to the conditions (2.4) and (2.5).

In the Minkowskian limit, corresponding to $\alpha \rightarrow \infty$ for fixed $y$, one has $z \approx \alpha+y$ and $z$ and $z_{j}$ are large. By using the asymptotic expressions for the modified Bessel functions for large values of the argument, we can see that

$$
\begin{aligned}
G_{\mu, \mu}\left(x z_{1}, x z\right) & \approx \frac{\sinh \left[x\left(y-y_{1}\right)\right]}{\alpha x}, \\
G_{\mu, \mu \pm 1}\left(x z_{1}, x z\right) & \approx \frac{\cosh \left[x\left(y-y_{1}\right)\right]}{\alpha x},
\end{aligned}
$$

and

$$
\Omega_{\nu}^{(j)}\left(x z_{1}, x z_{2}\right) \approx \frac{2 \alpha x}{e^{2 a x}-1} .
$$

Substituting these expressions in (5.2) and (5.8), to the leading order one gets $\left\langle F^{2}\right\rangle \rightarrow\left\langle F^{2}\right\rangle_{\mathrm{M}}, F=E, B$, with

$$
\begin{aligned}
\left\langle E^{2}\right\rangle_{\mathrm{M}}= & -\frac{(D-1) \Gamma((D+1) / 2)}{(4 \pi)^{(D-1) / 2} a^{D+1}} \\
& \times\left[\zeta(D+1) \mp \sum_{n=-\infty}^{\infty} \frac{3 / 2}{\left|n-\left(y-y_{1}\right) / a\right|^{D+1}}\right], \\
\left\langle B^{2}\right\rangle_{\mathrm{M}}= & -\frac{(D-1) \Gamma((D+1) / 2)}{(4 \pi)^{(D-1) / 2} a^{D+1}} \\
& \times\left[\zeta(D+1) \pm \sum_{n=-\infty}^{\infty} \frac{D-3 / 2}{\left|n-\left(y-y_{1}\right) / a\right|^{D+1}}\right]
\end{aligned}
$$

being the corresponding VEVs between two plates in the Minkowski bulk. Here, $\zeta(x)$ is the Riemann zeta function. By taking into account that $\zeta(4)=\pi^{4} / 90$, for $D=3$ from (5.16) we get

$$
\left\langle F^{2}\right\rangle_{\mathrm{M}}=\frac{1}{4 \pi a^{4}}\left[ \pm \sum_{n=-\infty}^{\infty} \frac{3 \delta_{F}}{\left|n-\left(y-y_{1}\right) / a\right|^{4}}-\frac{\pi^{4}}{45}\right] .
$$

Now, comparing with (5.13) we see that for $D=3$ the last term in (5.13) for the VEVs of the electric and magnetic fields squared is conformally related to the corresponding 
VEV in the region between two plates in the Minkowskian bulk. Near the plate $y=y_{1}$ the leading contribution in (5.16) comes from the term with $n=0$. As has been already mentioned, the corresponding leading term in the asymptotic expansion over the distance from the plate coincides with that for the Minkowski bulk [see (5.4) for the electric field].

The last terms in (5.2) and (5.8) are induced by adding the second plate to the geometry with a single plate at $z=z_{j}$. Let us consider the asymptotic behavior of the second-plate-induced parts. In the limit $z_{2} \rightarrow \infty$ for fixed $z$ and $z_{1}$ the dominant contribution to the integrals in (5.2) and (5.8) with $j=1$ comes from the region near the lower limit of the integration. By using the corresponding asymptotic expressions for the modified Bessel functions, for $\nu>0$ to the leading order we get

$$
\begin{aligned}
\left\langle F^{2}\right\rangle-\left\langle F^{2}\right\rangle_{1} \approx & \frac{\delta_{\nu}(D-1)\left(z / z_{2}\right)^{D+2 \nu}\left(z_{1} / z\right)^{2 \nu\left(1-\delta_{\nu}\right)}}{2^{D+2 \nu-3} \pi^{D / 2-1} \Gamma(D / 2+1) \Gamma^{2}(\nu) \alpha^{D+1}} \\
& \times\left[3-\delta_{\nu}-\left(1-\delta_{(F)}\right) D\right] \int_{0}^{\infty} d x x^{D+2 \nu-1} \frac{K_{\nu}(x)}{I_{\nu}(x)} .
\end{aligned}
$$

In the case $D=3$ the asymptotic is directly obtained from (5.11):

$$
\left\langle F^{2}\right\rangle-\left\langle F^{2}\right\rangle_{1} \approx-\frac{\pi^{3}\left(z / z_{2}\right)^{4}}{180 \alpha^{4}}\left(1-3 \delta_{\nu} \delta_{(F)}\right) .
$$

For $D=4$ and for the boundary condition (2.5) the corresponding asymptotic has the form

$$
\left\langle F^{2}\right\rangle-\left\langle F^{2}\right\rangle_{1} \approx-\frac{3 \delta_{(F)}\left(z / z_{2}\right)^{4}}{4 \pi \alpha^{5} \ln ^{2}\left(z_{1} / z_{2}\right)} \int_{0}^{\infty} d x x^{3} \frac{K_{0}(x)}{I_{0}(x)} .
$$

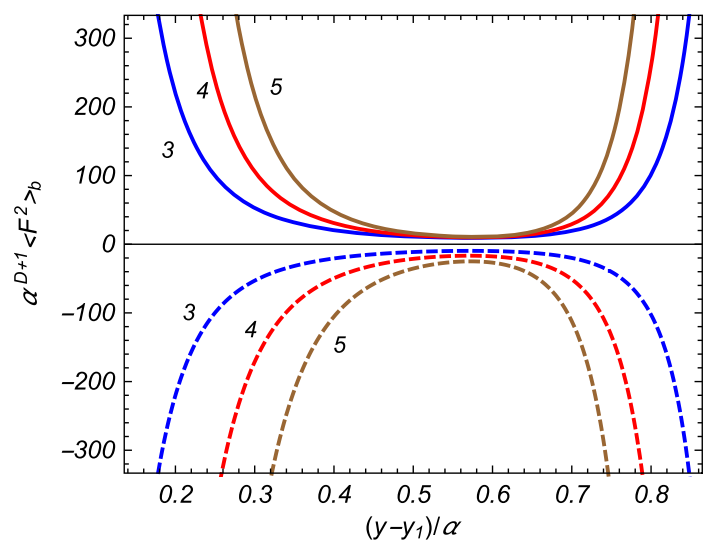

In terms of the physical distance of the observation point from the plate $z=z_{2}$, given by $y_{2}-y$, the second-plateinduced contributions decay as $\exp \left[-(D+2 \nu)\left(y_{2}-y\right) / \alpha\right]$ for $D \geq 5$, as $e^{-4\left(y_{2}-y\right) / \alpha} /\left(y_{2}-y\right)^{2}$ for $D=4$ and like $e^{-4\left(y_{2}-y\right) / \alpha}$ for $D=3$. In all these cases one has an exponential decay as a function of the distance. In the Minkowski bulk the decay of the second-plate-induced contributions is power law, like $1 /\left(y_{2}-y\right)^{D+1}$.

In the limit $z_{1} \rightarrow 0$, for fixed values $z$ and $z_{2}$, the corresponding asymptotics are obtained from (5.2) and (5.8) with $j=2$ by taking into account that for $\nu>0$ one has

$$
\Omega_{\nu}^{(2)}\left(x z_{1}, x z_{2}\right) \approx \frac{2 \nu\left(x z_{1} / 2\right)^{2 \nu}}{\Gamma^{2}(\nu+1) I_{\nu}^{2}\left(x z_{2}\right)} .
$$

As a consequence, the second-boundary-induced contributions $\left\langle E^{2}\right\rangle-\left\langle E^{2}\right\rangle_{2}$ and $\left\langle B^{2}\right\rangle-\left\langle B^{2}\right\rangle_{2}$ tend to zero like $\left(z_{1} / z_{2}\right)^{2 \nu}$. For $D=3$ the corresponding asymptotic expressions are obtained from (5.11) and $\left\langle F^{2}\right\rangle-\left\langle F^{2}\right\rangle_{2}$ decays as $z_{1} / z_{2}$. For $D=4$ and for the boundary condition (2.5) one has $\nu=0$ and the difference $\left\langle F^{2}\right\rangle-\left\langle F^{2}\right\rangle_{2}$ behaves as $1 / \ln \left(z_{1} / z\right)$.

In Fig. 1 we have plotted the boundary-induced parts $\left\langle F^{2}\right\rangle_{b}=\left\langle F^{2}\right\rangle-\left\langle F^{2}\right\rangle_{0}$ in the VEVs of the electric $(F=E)$ and magnetic $(F=B)$ fields squared in the region between the plates as functions of the distance from the plate at $z=z_{1}$ (in units of the curvature scale $\alpha$ ). The graphs are plotted for $a=\alpha$ and the full and dashed curves correspond to the electric and magnetic fields, respectively. The numbers near the curves are the corresponding values of the spatial dimension. The left and right panels are plotted for the boundary conditions (2.4) and (2.5), respectively. For the example presented in Fig. 1 the quantity $\left|\left\langle F^{2}\right\rangle_{b}\right|$ increases with increasing $D$. This is not a general feature. For example, in the case $a=2$ in the region near the point

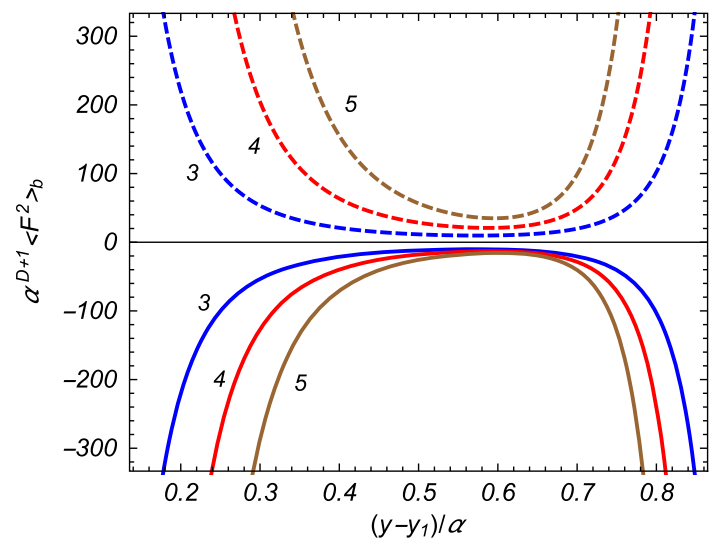

FIG. 1. The boundary-induced contributions in the VEVs of the electric (full curves) and magnetic (dashed curves) fields squared in the region between the plates as functions of the distance from the plate at $z=z_{1}$. The left and right panels correspond to the boundary conditions (2.4) and (2.5), respectively. The numbers near the curves are the values of the spatial dimension $D$. 
$y-y_{1}=a / 2$ the boundary-induced VEV $\left|\left\langle F^{2}\right\rangle_{b}\right|$ decreases with increasing $D$.

Having the correlators for the field strength tensor we can also evaluate the Casimir-Polder forces acting on a polarizable particle for the general case of anisotropic polarizability tensor and dispersion. In the static limit and for isotropic polarizability $\alpha_{\mathrm{P}}$, the Casimir-Polder potential is expressed in terms of the electric field squared as $U_{\mathrm{CP}}=-\alpha_{\mathrm{P}}\left\langle E^{2}\right\rangle / 2$. From the graphs in Fig. 1 it follows that in the region between the plates the Casimir-Polder forces near the plate at $z=z_{j}$ are attractive (repulsive) with respect to that plate for the boundary condition (2.4) [(2.5)]. At some intermediate point one has $\partial_{z}\left\langle E^{2}\right\rangle=0$ and the force vanishes. This equilibrium position of a polarizable particle is unstable for the condition (2.4) and stable for the condition (2.5).

\section{VEV OF THE ENERGY-MOMENTUM TENSOR}

Another important local characteristic of the vacuum state is the VEV of the energy-momentum tensor:

$$
\left\langle T_{\rho}^{\mu}\right\rangle=-\frac{1}{4 \pi}\left[\left\langle F_{\rho \kappa} F^{\mu \kappa}\right\rangle-\frac{1}{4} \delta_{\rho}^{\mu}\left\langle F_{\kappa \sigma} F^{\kappa \sigma}\right\rangle\right]
$$

By using the expressions (4.11) and (4.12) for the twopoint functions in the coincidence limit, we can see that the off-diagonal components vanish and for the diagonal components in the region $z_{1}<z<z_{2}$ we get

$$
\begin{aligned}
\left\langle T_{\rho}^{\mu}\right\rangle= & \left\langle T_{\rho}^{\mu}\right\rangle_{j}-\frac{\delta_{\nu} \delta_{\rho}^{\mu}(D-1) \alpha^{-1-D} z^{D+2}}{2(4 \pi)^{D / 2} \Gamma(D / 2+1)} \\
& \times \int_{0}^{\infty} d x x^{D+1} \Omega_{\nu}^{(j)}\left(x z_{1}, x z_{2}\right) \\
& \times\left[(D-4) G_{\nu, D / 2-1}^{2}\left(x z_{j}, x z\right)\right. \\
& \left.+(D-2) G_{\nu, D / 2-2}^{2}\left(x z_{j}, x z\right)\right]
\end{aligned}
$$

for $\mu, \rho=0, \ldots, D-1$ and

$$
\begin{aligned}
\left\langle T_{D}^{D}\right\rangle= & \left\langle T_{D}^{D}\right\rangle_{j}-\frac{\delta_{\nu}(D-1) \alpha^{-1-D} z^{D+2}}{(4 \pi)^{D / 2} \Gamma(D / 2)} \\
& \times \int_{0}^{\infty} d x x^{D+1} \Omega_{\nu}^{(j)}\left(x z_{1}, x z_{2}\right) \\
& \times\left[G_{\nu, D / 2-1}^{2}\left(x z_{j}, x z\right)-G_{\nu, D / 2-2}^{2}\left(x z_{j}, x z\right)\right] .
\end{aligned}
$$

The expressions (6.2) and (6.3) with $j=1$ and $j=2$ provide two equivalent representations of the VEVs. In these formulas, $\left\langle T_{\rho}^{\mu}\right\rangle_{j}$ is the VEV in the geometry of a single plate located at $z=z_{j}$. For $z>z_{j}$ one has

$$
\begin{aligned}
\left\langle T_{\rho}^{\mu}\right\rangle_{j}= & \left\langle T_{\rho}^{\mu}\right\rangle_{0}-\frac{\delta_{\nu} \delta_{\rho}^{\mu}(D-1) \alpha^{-1-D} z^{D+2}}{2(4 \pi)^{D / 2} \Gamma(D / 2+1)} \int_{0}^{\infty} d x x^{D+1} \frac{I_{\nu}\left(x z_{j}\right)}{K_{\nu}\left(x z_{j}\right)} \\
& \times\left[(D-4) K_{D / 2-1}^{2}(x z)+(D-2) K_{D / 2-2}^{2}(x z)\right], \\
\left\langle T_{D}^{D}\right\rangle_{j}= & \left\langle T_{D}^{D}\right\rangle_{0}-\frac{\delta_{\nu}(D-1) \alpha^{-1-D} z^{D+2}}{(4 \pi)^{D / 2} \Gamma(D / 2)} \int_{0}^{\infty} d x x^{D+1} \frac{I_{\nu}\left(x z_{j}\right)}{K_{\nu}\left(x z_{j}\right)} \\
& \times\left[K_{D / 2-1}^{2}(x z)-K_{D / 2-2}^{2}(x z)\right],
\end{aligned}
$$

with $\nu$ given by (2.9). In the region $z<z_{j}$ the VEVs $\left\langle T_{\nu}^{\mu}\right\rangle_{j}$ are obtained from (6.4) by the replacements $I \rightleftarrows K$ of the modified Bessel functions in the boundary-induced contributions. In the region $z<z_{1}$ the VEV of the energymomentum tensor is given by (6.4) with $j=1$ and with the replacements $I \rightleftarrows K$. The VEV in the region $z>z_{2}$ is given by (6.4) with $j=2$. The boundary-induced VEVs in the geometry of a single plate and for the boundary condition (2.4) have been previously investigated in [19-22]. For the same boundary condition, a part of the results concerning the VEV of the energy-momentum tensor in the region between the plates has been presented at the Tenth Alexander Friedmann International Conference [29]. As seen from (6.2), the components $\left\langle T_{\rho}^{\mu}\right\rangle$ with $\mu, \rho=0,1, \ldots, D-1$, coincide. This is a consequence of the Poincare invariance of the problem in the subspace $\left(x^{0}, x^{1}, \ldots, x^{D-1}\right)$. In the expressions (6.2)-(6.4) and for points away from the plates, the renormalization is required for the boundary-free contribution $\left\langle T_{\rho}^{\mu}\right\rangle_{0}$ only. From the maximal symmetry of the bulk geometry we expect that the corresponding renormalized VEV has the form $\left\langle T_{\rho}^{\mu}\right\rangle_{0}=$ const $\cdot \delta_{\rho}^{\mu}$ and it does not depend on spacetime point.

We will denote by $\left\langle T_{\rho}^{\mu}\right\rangle_{\mathrm{b}}=\left\langle T_{\rho}^{\mu}\right\rangle-\left\langle T_{\rho}^{\mu}\right\rangle_{0}$ the boundaryinduced contribution to the VEV of the energy-momentum tensor. One can check that it obeys the covariant continuity equation $\nabla_{\mu}\left\langle T_{\rho}^{\mu}\right\rangle_{\mathrm{b}}=0$. In the problem under consideration the latter is reduced to the relation

$$
z^{D+1} \partial_{z}\left(z^{-D}\left\langle T_{D}^{D}\right\rangle_{\mathrm{b}}\right)+D\left\langle T_{0}^{0}\right\rangle_{\mathrm{b}}=0
$$

between the energy density and the normal stress. For $D>3$ the boundary-induced contribution in the vacuum energy density is negative for the boundary condition (2.4) and positive for the boundary condition (2.5) for all values of $z$, including the regions $\left.z<z_{1}, z\right\rangle z_{2}$. Near the plate $z=z_{j}$ and for the boundary condition (2.4) [(2.5)], the boundary-induced contribution to the normal stress $\left\langle T_{D}^{D}\right\rangle$ is negative (positive) in the region $z>z_{j}$ and positive (negative) in the region $z<z_{j}$. The trace of the boundary-induced contribution in the VEV of the energymomentum tensor is related to the corresponding photon condensate by the formula

$$
\left\langle T_{\mu}^{\mu}\right\rangle_{\mathrm{b}}=-\frac{D-3}{16 \pi}\left\langle F_{\mu \sigma} F^{\mu \sigma}\right\rangle_{\mathrm{b}}
$$


For $D=3$ that contribution is traceless. The latter property is a consequence of the conformal invariance of the electromagnetic field in $D=3$. Note that because of the conformal anomaly the boundary-free part has nonzero trace, $\left\langle T_{\mu}^{\mu}\right\rangle_{0} \neq 0$.

For $D>3$ the VEVs diverge on the plates. The divergence on the plate $z=z_{j}$ comes from the single boundary part $\left\langle T_{\rho}^{\mu}\right\rangle_{j}$. The leading terms in the asymptotic expansion over the distance from the plate are expressed as

$$
\begin{aligned}
& \left\langle T_{0}^{0}\right\rangle \approx-\frac{D-3}{12 \pi}\left\langle E^{2}\right\rangle, \\
& \left\langle T_{D}^{D}\right\rangle \approx\left(z / z_{j}-1\right)\left\langle T_{0}^{0}\right\rangle \approx \frac{y-y_{j}}{\alpha}\left\langle T_{0}^{0}\right\rangle,
\end{aligned}
$$

with $\left\langle E^{2}\right\rangle$ given by (5.4). This type of divergence is well known in quantum field theory with boundaries. In (6.2) and (6.3) the second-plate-induced contributions (the second terms in the right-hand sides) are finite at $z=z_{j}$.

In the special case $D=3$, by using the expressions for the functions $I_{ \pm 1 / 2}(u), K_{ \pm 1 / 2}(u)$, and (5.10) we can see that in the region between the plates

$$
\begin{aligned}
& \left\langle T_{\rho}^{\mu}\right\rangle=\left\langle T_{\rho}^{\mu}\right\rangle_{0}-\frac{\delta_{\rho}^{\mu} \pi^{2}(z / \alpha)^{4}}{720\left(z_{2}-z_{1}\right)^{4}}, \\
& \left\langle T_{3}^{3}\right\rangle=\left\langle T_{3}^{3}\right\rangle_{0}+\frac{\pi^{2}(z / \alpha)^{4}}{240\left(z_{2}-z_{1}\right)^{4}},
\end{aligned}
$$

where $\mu \neq 3$. Note that the boundary-induced contributions (the second terms in the right-hand sides) in these expressions are the same for the boundary conditions (2.4) and (2.5). Those contributions are conformally related to the corresponding VEVs in the region between two plates in the Minkowski bulk with the conformal factor $(z / \alpha)^{4}$. In the region $z>z_{2}$ one has $\left\langle T_{\rho}^{\mu}\right\rangle=\left\langle T_{\rho}^{\mu}\right\rangle_{0}$. In the region $z<z_{1}$ the VEVs for the boundary condition (2.4) are given by the expressions $(\mu, \rho=0,1,2)$

$$
\begin{aligned}
& \left\langle T_{\rho}^{\mu}\right\rangle=\left\langle T_{\rho}^{\mu}\right\rangle_{0}-\frac{\delta_{\rho}^{\mu} \pi^{2}}{720}\left(\frac{z}{\alpha z_{1}}\right)^{4}, \\
& \left\langle T_{3}^{3}\right\rangle=\left\langle T_{3}^{3}\right\rangle_{0}+\frac{\pi^{2}}{240}\left(\frac{z}{\alpha z_{1}}\right)^{4} .
\end{aligned}
$$

From (6.9) it follows that the $z$ projection of Casimir force acting per unit surface of the plate at $z=z_{1}$ (from the side $z=z_{1}-0$ ) is given by $-\pi^{2} \alpha^{-4} / 240$. The latter does not depend on the location of the plate and is directed toward the AdS boundary. For the boundary condition (2.5) the expressions for VEVs in the region $z<z_{1}$ read

$$
\begin{aligned}
& \left\langle T_{\rho}^{\mu}\right\rangle=\left\langle T_{\rho}^{\mu}\right\rangle_{0}+\delta_{\rho}^{\mu} \frac{7 \pi^{2}}{5760}\left(\frac{z}{\alpha z_{1}}\right)^{4}, \\
& \left\langle T_{3}^{3}\right\rangle=\left\langle T_{3}^{3}\right\rangle_{0}-\frac{7 \pi^{2}}{1920}\left(\frac{z}{\alpha z_{1}}\right)^{4},
\end{aligned}
$$

with $\mu=0,1,2$. Note that (6.10) differs from the corresponding result for the boundary condition (2.4) [given by (6.9)]. The corresponding energy density is positive and the $z$ projection of Casimir force acting per unit surface of the side $z=z_{1}-0$ is given by $7 \pi^{2} \alpha^{-4} / 1920$. The latter is repulsive with respect to the AdS boundary.

In the Minkowskian limit $\alpha \rightarrow \infty$ for fixed $y$ and $y_{j}$, by using the relations (5.15), to the leading order we get $\left\langle T_{\rho}^{\mu}\right\rangle \rightarrow\left\langle T_{\rho}^{\mu}\right\rangle_{\mathrm{M}}$, with the Minkowskian VEVs

$$
\begin{aligned}
\left\langle T_{\rho}^{\mu}\right\rangle_{\mathrm{M}}= & -\delta_{\rho}^{\mu} \frac{(D-1) \Gamma((D+1) / 2)}{(4 \pi)^{(D+1) / 2} a^{D+1}} \\
& \times\left[\zeta(D+1) \pm \sum_{n=-\infty}^{\infty} \frac{(D-3) / 2}{\left|n-\left(y-y_{1}\right) / a\right|^{D+1}}\right] \\
\left\langle T_{D}^{D}\right\rangle_{\mathrm{M}}= & \frac{D(D-1) \Gamma((D+1) / 2)}{(4 \pi)^{(D+1) / 2} a^{D+1}} \zeta(D+1),
\end{aligned}
$$

where $\mu=0,1, \ldots, D-1$ and the upper and lower signs correspond to the boundary conditions (2.4) and (2.5), respectively. For $D=3$ these results are conformally related to the boundary-induced VEVs in the AdS bulk [see (6.8)]. Note that the normal stress is uniform and is the same for the conditions (2.4) and (2.5).

The last terms in (6.2) and (6.3) are the contribution induced by the second plate when we add it to the geometry with a single plate located at $z=z_{j}$. Let us consider the corresponding asymptotics for limiting values of the second plate location. In the limit $z_{2} \rightarrow \infty$, when $z$ and $z_{1}$ are fixed, for $D>4$ from (6.2) and (6.3) with $j=1$ we find

$$
\begin{aligned}
\left\langle T_{D}^{D}\right\rangle-\left\langle T_{D}^{D}\right\rangle_{1} \approx & -\delta_{\nu} D \frac{\left\langle T_{0}^{0}\right\rangle-\left\langle T_{0}^{0}\right\rangle_{1}}{D-3+\delta_{\nu}} \\
\approx & \frac{(D-1) \alpha^{-1-D}\left(z / z_{2}\right)^{D+2 \nu}}{2^{D+2 \nu-2} \pi^{D / 2} \Gamma(D / 2) \Gamma^{2}(\nu)}\left(z_{1} / z\right)^{2 \nu\left(1-\delta_{\nu}\right)} \\
& \times \int_{0}^{\infty} d x x^{D+2 \nu-1} \frac{K_{\nu}(x)}{I_{\nu}(x)}
\end{aligned}
$$

and the second plate contributions decay as $\left(z / z_{2}\right)^{D+2 \nu}$. Note that for the boundary condition (2.4) the leading term does not depend on $z_{1}$. For $D=4$, the leading terms in the limit $z_{2} \rightarrow \infty$ are given by 


$$
\begin{aligned}
\left\langle T_{D}^{D}\right\rangle-\left\langle T_{D}^{D}\right\rangle_{1} & \approx-2\left(\left\langle T_{0}^{0}\right\rangle-\left\langle T_{0}^{0}\right\rangle_{1}\right) \\
& \approx \frac{3 \alpha^{-5}\left(z_{1} / z_{2}\right)^{4}}{16 \pi^{2} \ln ^{2}\left(z_{1} / z_{2}\right)}\left(z / z_{1}\right)^{6} \int_{0}^{\infty} d x x^{3} \frac{K_{0}(x)}{I_{0}(x)} .
\end{aligned}
$$

The asymptotic for $D=3$ is directly obtained from (6.8). The leading terms in the limit $z_{1} \rightarrow 0$, for fixed $z$ and $z_{2}$, are obtained from (6.2) and (6.3) with $j=2$ by the replacement (5.21) and the second-plate-induced contributions vanish like $\left(z_{1} / z_{2}\right)^{D-2}$.

Figure 2 presents the boundary-induced contributions in the VEVs of the energy density ( $\mu=0$, full curves) and the normal stress ( $\mu=D$, dashed curves) in the region between the plates with the separation $a / \alpha=1$. The left and right panels correspond to the boundary conditions (2.4) and (2.5), respectively. For $D=3$ the VEVs are finite on the boundaries and in the region between the plates they coincide for the conditions (2.4) and (2.5).

\section{THE CASIMIR FORCES}

The Casimir force acting on the plate is determined by the normal stress $\left\langle T_{D}^{D}\right\rangle$ evaluated at the location of the plate. The boundary-free contributions to the normal stress are the same on the left- and right-hand sides of the plate and will not contribute to the net force. For the plate at $z=z_{j}$, the remaining contribution to the Casimir force per unit surface is decomposed into two parts:

$$
p_{j}=p_{j}^{(\mathrm{s})}+p_{j}^{(\mathrm{int})},
$$

where $p_{j}^{(\mathrm{s})}$ is the vacuum pressure on the plate at $z=z_{j}$ when the second plate is absent (self-action force) and the part $p_{j}^{\text {(int) }}$ is induced by the second plate (interaction force). The expression for $p_{j}^{(\mathrm{s})}$ is obtained by combining the vacuum pressures on the left- and right-hand sides of the

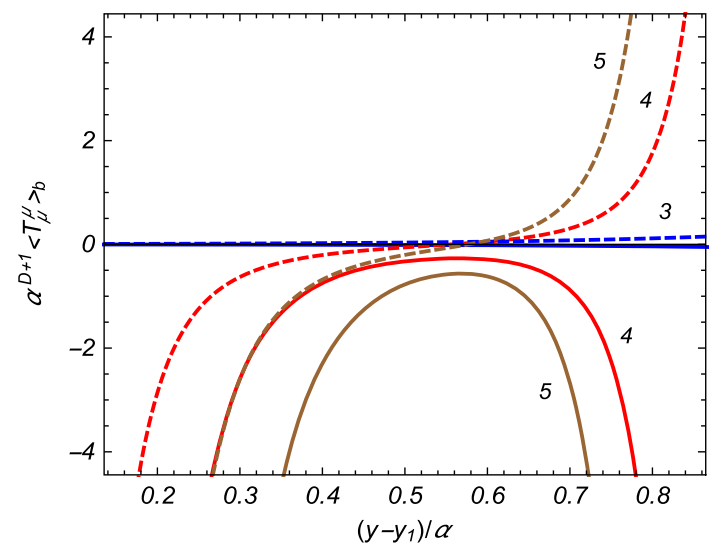

plate. The $z$ projection of the self-action force acting per unit surface of the plate at $z=z_{j}$ is given by $f_{j}^{(\mathrm{s})}=\left.\left\langle T_{D}^{D}\right\rangle_{j}\right|_{z=z_{j}-0} ^{z=z_{j}+0}$. For $D>3$ the self-action contributions are divergent and they require an additional renormalization. For $D=3$ and for the boundary condition (2.4) one has $\left\langle T_{D}^{D}\right\rangle_{j}=\left\langle T_{D}^{D}\right\rangle_{0}$ for $z=z_{j}+0$ and $\left\langle T_{D}^{D}\right\rangle_{j}=$ $\left\langle T_{D}^{D}\right\rangle_{0}+\pi^{2} \alpha^{-4} / 240$ for $z=z_{j}-0$. Hence, in this case one gets

$$
f_{j}^{(s)}=-\frac{\pi^{2}}{240 \alpha^{4}},
$$

and the self-action force is directed toward the AdS boundary. In a similar way, for the boundary condition (2.5) and for $D=3$ we find

$$
f_{j}^{(s)}=\frac{7 \pi^{2}}{1920 \alpha^{4}} .
$$

For this case the force is directed from the AdS boundary. Note that in both cases of the boundary conditions the selfaction force per unit surface do not depend on the location of the plate.

In contrast to the self-action part, the interaction term $p_{j}^{\text {(int) }}$ is finite and does not require a further renormalization. By using the expression (6.3) for the normal stress, one finds

$$
\begin{aligned}
& p_{1}^{\text {(int) }}=-\frac{(D-1) \alpha^{-1-D}}{2^{D} \pi^{D / 2} \Gamma(D / 2)} \int_{0}^{\infty} d x \frac{x^{D-1} K_{\nu}(x \eta)}{K_{\nu}(x) G_{\nu, \nu}(x, x \eta)}, \\
& p_{2}^{\text {(int) }}=-\frac{(D-1) \alpha^{-1-D}}{2^{D} \pi^{D / 2} \Gamma(D / 2)} \int_{0}^{\infty} d x \frac{x^{D-1} I_{\nu}(x / \eta)}{I_{\nu}(x) G_{\nu, \nu}(x / \eta, x)},
\end{aligned}
$$

where $\eta$ is defined as (2.17). Here, $p_{1}^{\text {(int) }}$ acts on the side $z=z_{1}+0$ for the plate at $z=z_{1}$ and $p_{2}^{\text {(int) }}$ acts on the side $z=z_{2}-0$ for the plate at $z=z_{2}$. The $z$ projection of the

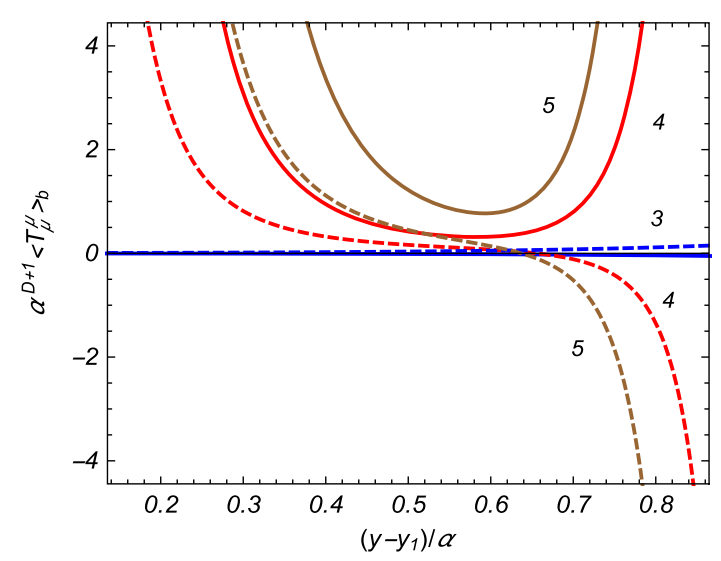

FIG. 2. The boundary-induced parts in the VEVs of the energy density (full curves) and the normal stress (dashed curves) versus the distance from the plate at $z=z_{1}$. The left and right panels present the graphs for the boundary conditions (2.4) and (2.5), respectively. The numbers near the curves correspond to the values of $D$. 
corresponding force acting per unit surface of the plate at $z=z_{j}$ is given by $f_{j}^{(\mathrm{int})}=(-1)^{j} p_{j}^{(\mathrm{int})}$. By taking into account that $G_{\nu, \nu}(u, v)>0$ for $v>u$, we see that $p_{j}^{(\text {int })}<0$ and the interaction forces between the plates are always attractive. As is seen, the interaction terms depend on the locations of the plates through the ratio $z_{2} / z_{1}$. This is a consequence of the maximal symmetry of the AdS spacetime. Note that the integrand in (7.4) for $p_{j}^{\text {(int) }}$ is also expressed as $x^{D-1} \Omega_{\nu}^{(j)}(x, x \eta)$. By taking into account that

$\Omega_{\nu}^{(j)}\left(u z_{1}, u z_{2}\right)=(-1)^{j} z_{j} \partial_{z_{j}} \ln \left|1-\frac{I_{\nu}\left(u z_{1}\right) K_{\nu}\left(u z_{2}\right)}{I_{\nu}\left(u z_{2}\right) K_{\nu}\left(u z_{1}\right)}\right|$,

the $z$ projection of the interaction force is presented in an alternative form:

$$
\begin{aligned}
f_{j}^{(\text {int })}= & -\frac{(D-1) \alpha^{-1-D} z_{j}^{D+1}}{2^{D} \pi^{D / 2} \Gamma(D / 2)} \\
& \times \partial_{z_{j}} \int_{0}^{\infty} d u u^{D-1} \ln \left|1-\frac{I_{\nu}\left(u z_{1}\right) K_{\nu}\left(u z_{2}\right)}{I_{\nu}\left(u z_{2}\right) K_{\nu}\left(u z_{1}\right)}\right| .
\end{aligned}
$$

In the Minkowskian limit, $\alpha \rightarrow \infty$, by using the asymptotic expressions (5.15), we can see that $p_{j}^{(\text {int) }} \rightarrow p_{\mathrm{M}}$, where the Casimir pressure in the Minkowski bulk is given by

$$
p_{\mathrm{M}}=-D(D-1) \frac{\Gamma((D+1) / 2) \zeta(D+1)}{(4 \pi)^{(D+1) / 2} a^{D+1}} .
$$

Note that the self-action stresses $\left.\left\langle T_{D}^{D}\right\rangle_{j}\right|_{z=z_{j}-0}$ and $\left.\left\langle T_{D}^{D}\right\rangle_{j}\right|_{z=z_{j}+0}$ on a single plate in the Minkowski bulk are equal and the corresponding net force vanishes. Another special case corresponds to $D=3$ with general $\alpha$. The interaction parts are obtained from (7.4). The $z$ projection of the total force acting per unit surface of the plate at $z=z_{j}$ is expressed as

$$
f_{j}=f_{j}^{(\mathrm{s})}+(-1)^{j} p_{j}^{(\mathrm{int})} .
$$

By taking into account the expressions (7.2) and (7.3) for $f_{j}^{(\mathrm{s})}$, for the boundary condition (2.4) one finds

$$
\begin{aligned}
& f_{1}=-\frac{\pi^{2}}{240 \alpha^{4}}\left[1-\left(e^{a / \alpha}-1\right)^{-4}\right], \\
& f_{2}=-\frac{\pi^{2}}{240 \alpha^{4}}\left(1-e^{-a / \alpha}\right)^{-4},
\end{aligned}
$$

where $a / \alpha$ is the proper distance between the plates measured in units of the AdS curvature scale $\alpha$. For the boundary condition (2.5) $f_{2}$ coincides with (7.9) and for $f_{1}$ we get

$$
f_{1}=\frac{\pi^{2}}{240 \alpha^{4}}\left[\frac{7}{8}+\left(e^{a / \alpha}-1\right)^{-4}\right] .
$$

The difference of the forces $f_{1}$ for the boundary conditions (2.4) and (2.5) is a consequence of different interactions of the plate at $z=z_{1}$ with the AdS boundary. In Fig. 3 we have plotted the forces $f_{j}$ from (7.9) and (7.10) as functions of $a / \alpha$. Curve 1 corresponds to the force $f_{1}$ for the condition (2.4), curve 2 presents the force $f_{2}$ (is the same for both the boundary conditions), and curve 3 corresponds to the force $f_{1}$ for the condition (2.5). As seen from the graphs, the Casimir force on the plate at $z=z_{2}$ is always directed toward the AdS boundary for both boundary conditions. For the boundary condition (2.5) the force on the plate at $z=z_{1}$ is directed toward the AdS horizon. In the case of the boundary condition (2.4) the force acting on the plate at $z=z_{1}$ is directed toward the AdS horizon for small separations between the plates and toward the AdS boundary for large separations. At $a / \alpha \approx 0.693$ that force becomes zero.

Now let us consider the asymptotics of the interaction forces at small and large separations between the plates. For proper distances much smaller than the AdS curvature scale one has $a / \alpha \ll 1$ and $z_{2} / z_{1}-1 \ll 1$. In this limit the dominant contribution to the integrals in (7.4) comes from large values of $x$. By using the asymptotic expressions for the modified Bessel functions for large arguments we can see that to the leading order $p_{1}^{\text {(int) }} \approx p_{2}^{\text {(int) }} \approx p_{\mathrm{M}}$, where the vacuum pressures in the Minkowski bulk are given by (7.7). This shows that for small separations the effect of gravity on the Casimir forces is weak. This is related to the fact that at such separations the dominant contribution to the forces come from the vacuum fluctuations with wavelengths

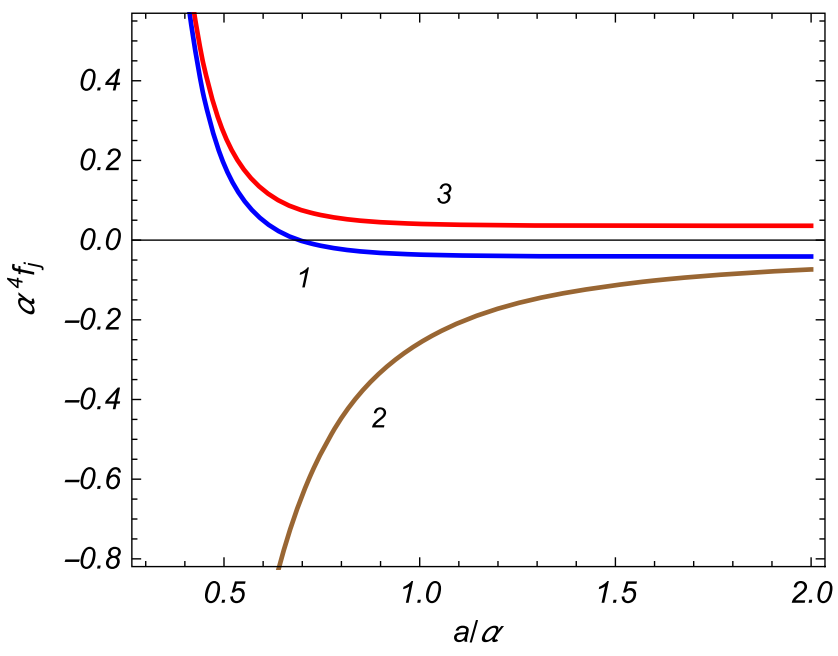

FIG. 3. The $z$ projections of the Casimir forces for $D=3$ as functions of the separation between the plates. Curves 1 and 3 correspond to the force $f_{1}$ for the boundary conditions (2.4) and (2.5), respectively. Curve 2 correspond to the force $f_{2}$ [it coincides for the conditions (2.4) and (2.5)]. 
smaller than the curvature radius. The influence of the gravitational field on these fluctuations is weak. At large separations between the plates one has $a / \alpha \gg 1$ and $z_{2} / z_{1} \gg 1$. For $D=3$ the corresponding asymptotics are obtained from (7.9) and (7.10). For $D>3$ from (7.4) to the leading order we find

$$
\begin{aligned}
& p_{1}^{(\mathrm{int})} \approx-\frac{4(D-1) \alpha^{-1-D} e^{-(D+2 \nu) a / \alpha}}{2^{D+2 \nu} \pi^{D / 2} \Gamma(D / 2) \Gamma^{2}(\nu)} \int_{0}^{\infty} d x x^{D+2 \nu-1} \frac{K_{\nu}(x)}{I_{\nu}(x)}, \\
& p_{2}^{(\mathrm{int})} \approx-\frac{2 \nu(D-1) \alpha^{-1-D} e^{-2 \nu a / \alpha}}{2^{D+2 \nu} \pi^{D / 2} \Gamma(D / 2) \Gamma^{2}(\nu+1)} \int_{0}^{\infty} d x \frac{x^{D+2 \nu-1}}{I_{\nu}^{2}(x)} .
\end{aligned}
$$

The case $D=4$ for the boundary condition (2.5) should be considered separately:

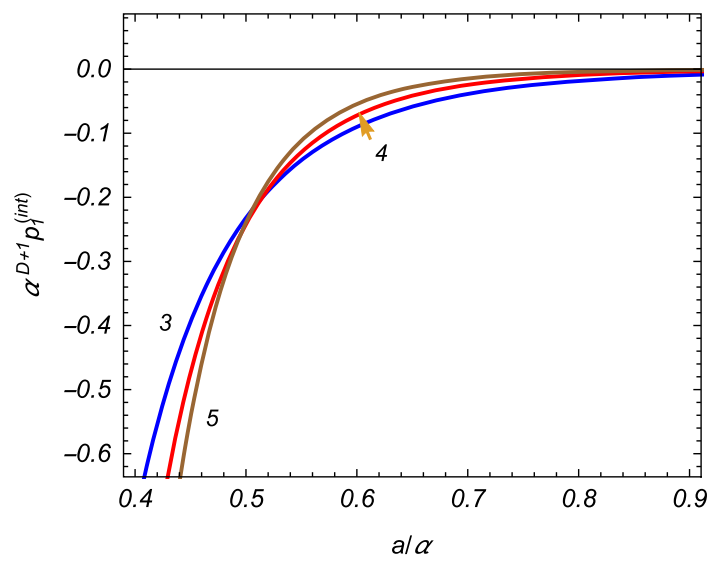

$$
\begin{aligned}
& p_{1}^{\text {(int) }} \approx-\frac{3 e^{-4 a / \alpha}}{16 \pi^{2} \alpha^{3} a^{2}} \int_{0}^{\infty} d x x^{3} \frac{K_{0}(x)}{I_{0}(x)}, \\
& p_{2}^{\text {(int) }} \approx-\frac{3 \alpha^{-4}}{16 \pi^{2} a} \int_{0}^{\infty} d x \frac{x^{3}}{I_{0}^{2}(x)} .
\end{aligned}
$$

The integrals in (7.12) are equal to 1.0045 and 4.0181 for $p_{1}^{\text {(int) }}$ and $p_{2}^{\text {(int) }}$, respectively. From (7.11) we see that for confining boundary conditions (2.5) the decay of the interaction forces is weaker. For both the boundary conditions (2.4) and (2.5) at large separations the interaction parts are exponentially suppressed as functions of the separation $a$. This behavior is in clear contrast with that for the Minkowski bulk where the Casimir forces decay as $1 / a^{D+1}$ for all separations between the plates.

In Figs. 4 and 5 we display the interaction pressures (7.4) versus the separation between the plates (in units of the curvature radius) for different values of the spatial

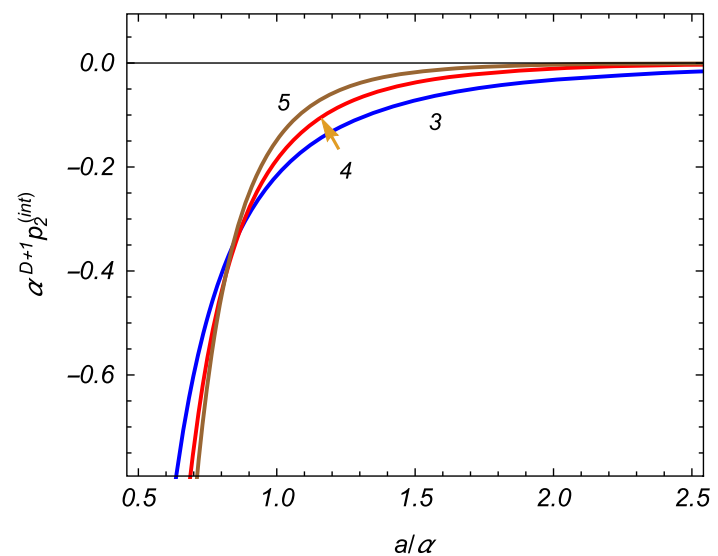

FIG. 4. The interaction parts in the vacuum pressures on the plates for the boundary condition (2.4) as functions of the separation. The left and right panels correspond to the forces acting on the plates $z=z_{1}$ and $z=z_{2}$, respectively, and the numbers near the curves are the values of the spatial dimension $D$.
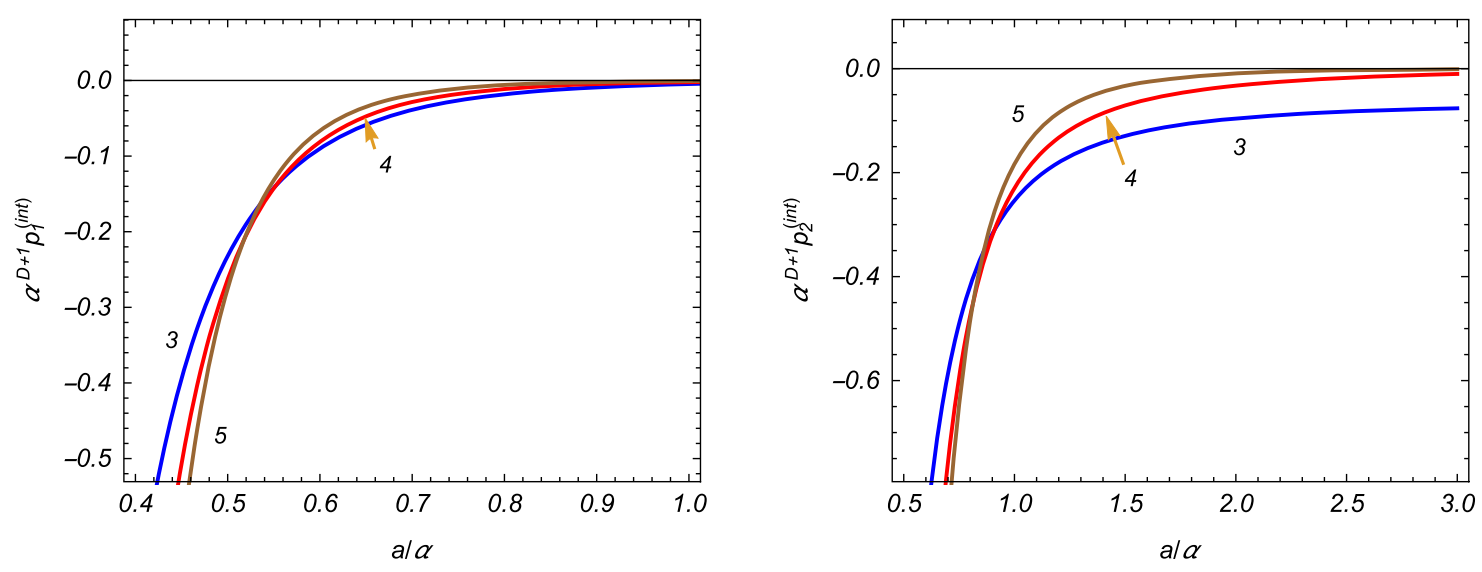

FIG. 5. The same as in Fig. 4 for the boundary condition (2.5). 
dimension $D$ (numbers near the curves). The left and right panels correspond to the forces acting on the plates $z=z_{1}$ and $z=z_{2}$, respectively. Figure 4 is plotted for the boundary condition (2.4), and Fig. 5 presents the results for the condition (2.5).

\section{VACUUM ENERGY}

In the previous sections we have considered the local characteristics of the vacuum and the Casimir forces acting on the boundaries. Here we are interested in the total vacuum energy and its relation to the Casimir forces.

\section{A. Zeta function and the vacuum energy in the region between the plates}

In the region between the plates the corresponding eigenvalues of the quantum number $\lambda$ are solutions of Eq. (2.12) where $\nu$ is defined as (2.9) and the function $g_{\nu, \rho}(x, y)$ is given by (2.11). First let us consider the expression for the VEV of the energy density based on the representation (3.4) for the two-point function. By using (6.1) we get

$$
\begin{aligned}
\left\langle T_{0}^{0}\right\rangle= & \frac{(D-1) \alpha^{-1-D} z^{D+2}}{16(2 \pi)^{D-3} z_{1}^{2}} \int d \mathbf{k} \sum_{n=1}^{\infty} \frac{u}{\omega_{\nu, n}} T_{\nu}(\eta, u) \\
& \times\left\{\omega_{\nu, n}^{2} g_{\nu, D / 2-1}^{2}\left(u, u z / z_{1}\right)\right. \\
& +\left(\frac{k^{2}}{D-1}+\frac{u^{2}}{2 z_{1}^{2}}\right)\left[g_{\nu, D / 2-2}^{2}\left(u, u z / z_{1}\right)\right. \\
& \left.\left.-g_{\nu, D / 2-1}^{2}\left(u, u z / z_{1}\right)\right]\right\}_{u=\lambda_{\nu, n}},
\end{aligned}
$$

where $\omega_{\nu, n}$ is given by (2.13).

By taking into account the boundary conditions (2.12) and making use of the integral for the square of cylinder functions, it can be seen that

$$
\begin{aligned}
\int_{z_{1}}^{z_{2}} d z z g_{\nu, D / 2-1}^{2}\left(u, u z / z_{1}\right) & =\int_{z_{1}}^{z_{2}} d z z g_{\nu, D / 2-2}^{2}\left(u, u z / z_{1}\right) \\
& =\frac{2 z_{1}^{2}}{\pi^{2} u T_{\nu}(\eta, u)}
\end{aligned}
$$

where $u=\lambda_{\nu, n}$. Combining this with (8.1) the following relation is obtained:

$$
\mathcal{E}_{(\mathrm{II})}=\int_{z_{1}}^{z_{2}} d z\left(\frac{\alpha}{z}\right)^{D+1}\left\langle T_{0}^{0}\right\rangle
$$

Here

$$
\begin{aligned}
\mathcal{E}_{(\mathrm{II})} & =\frac{1}{2} \sum_{\sigma} \int \frac{d \mathbf{k}}{(2 \pi)^{D-1}} \sum_{n=1}^{\infty} \omega_{\nu, n} \\
& =\frac{D-1}{2} \int \frac{d \mathbf{k}}{(2 \pi)^{D-1}} \sum_{n=1}^{\infty} \sqrt{\lambda_{\nu, n}^{2} / z_{1}+k^{2}}
\end{aligned}
$$

is the total vacuum energy in the region $z_{1} \leq z \leq z_{2}$ (region II) per unit coordinate volume along the parallel directions $\left(x^{1}, \ldots, x^{D-1}\right)$. The factor $D-1$ in the second expression is the number of independent polarizations of the electromagnetic field in $D$-dimensional space. Hence, we have seen that the integral of the bulk energy density is equal to the vacuum energy evaluated as the sum of the ground state energies of elementary oscillators. Note that these two quantities, in general, can be different. The difference may be related to the surface energy density located on the boundaries. An example of this kind of problem is provided by a scalar field with a Robin boundary condition [30]. The corresponding surface energy-momentum tensor for general bulk and boundary geometries has been considered in [31]. The VEV of the surface energy density and the energy balance in braneworld models on the AdS bulk were discussed in [32].

For the regularization of the divergent expression in the right-hand side of (8.3) we use the generalized zeta function method [33]. This method has been widely used in the evaluation of the Casimir energy for various bulk and boundary geometries [1], in particular, in braneworld models [6-9]. Let us introduce the zeta function related to $(8.3)$ :

$\zeta(s)=\frac{(D-1)(4 \pi)^{(1-D) / 2}}{\Gamma((D-1) / 2)} \mu^{s+1} \sum_{n=1}^{\infty} \int_{0}^{\infty} d k \frac{k^{D-2}}{\left(\lambda_{\nu, n}^{2} / z_{1}^{2}+k^{2}\right)^{s / 2}}$,

considered as a function of the complex variable $s$. The parameter $\mu$ has dimension of mass and is introduced to keep the dimension of the right-hand side. For the evaluation of the vacuum energy we need the analytical continuation of $\zeta(s)$ at the point $s=-1$. After the integration over $k,(8.4)$ is presented in terms of the partial zeta function $\zeta_{p}(s)=\sum_{n=1}^{\infty} \lambda_{\nu, n}^{-s}$. The corresponding formula reads

$$
\zeta(s)=\frac{(D-1) \Gamma((s+1-D) / 2)}{2(4 \pi)^{(D-1) / 2} \Gamma(s / 2) z_{1}^{D}}\left(\mu z_{1}\right)^{s+1} \zeta_{p}(s+1-D) .
$$

Hence, the problem is reduced to the analytic continuation of the partial zeta function $\zeta_{p}(s)$ at the point $s=-D$.

In the cases $\nu= \pm 1 / 2$ the function $g_{\nu, \nu}\left(\lambda z_{1}, \lambda z\right)$ is given by (2.14) and the eigenvalues $\lambda_{\nu, n}$ are simplified to (2.15). The corresponding partial zeta function $\zeta_{p}(s)$ is expressed 
in terms of the Riemann zeta function $\zeta_{R}(x)$. For the function (8.5) one finds

$$
\zeta(s)=\frac{(D-1) \Gamma((D-s) / 2) \mu^{s+1}}{2^{D} \pi^{D / 2} \Gamma(s / 2)\left(z_{2}-z_{1}\right)^{D-1-s}} \zeta_{R}(D-s) .
$$

In deriving this expression we have used the relation

$$
\pi^{x+1 / 2} \Gamma(-x / 2) \zeta_{R}(-x)=\Gamma((x+1) / 2) \zeta_{R}(x+1)
$$

for the product of the gamma and Riemann zeta functions. The expression (8.6) is finite at the physical point $s=-1$ and for the vacuum energy we get

$$
\mathcal{E}_{(\mathrm{II})}=-(D-1) \frac{\Gamma((D+1) / 2) \zeta_{R}(D+1)}{(4 \pi)^{(D+1) / 2}\left(z_{2}-z_{1}\right)^{D}} .
$$

The special cases under consideration are realized for $D=3$ :

$$
\mathcal{E}_{(\mathrm{II})}=-\frac{\pi^{2}}{720\left(z_{2}-z_{1}\right)^{3}}
$$

for both the boundary conditions (2.4) and (2.5). This coincides with the $D=3$ Casimir energy for plates in the Minkowski bulk with separation $z_{2}-z_{1}$. In the case of boundary condition (2.5) one has $\nu=1 / 2$ for $D=5$ and the corresponding energy is given by

$$
\mathcal{E}_{(\mathrm{II})}=-\frac{\pi^{3}}{7560\left(z_{2}-z_{1}\right)^{5}} .
$$

Now we return to the general case of $\nu$. The partial zeta function is presented as the contour integral

$$
\zeta_{p}(s)=\frac{1}{2 \pi i} \int_{C} \frac{d u}{u^{s}} \partial_{u} \ln \left[g_{\nu, \nu}(u, u \eta)\right]
$$

in the complex plane $u$. The closed counterclockwise contour $C$ consists of large semicircle $C_{R}$ in the right half-plane, with the center at $u=0$ and with the radius $R$ tending to infinity, and a straight part that coincides with the imaginary axis. The point $u=0$ is avoided by semicircle $C_{r}$ in the right half-plane with small radius $r$. For $\operatorname{Re} s<2$ the contribution from the latter contour to the integral in (8.11) vanishes in the limit $r \rightarrow 0$. The integral is decomposed as

$$
\begin{aligned}
\zeta_{p}(s)= & \frac{\eta^{s}}{2 \pi i} \int_{C} \frac{d u}{u^{s}} \partial_{u} \ln \left[u^{-\nu} J_{\nu}(u)\right] \\
& +\frac{1}{2 \pi i} \sum_{j=1,2} \int_{C_{j}} \frac{d u}{u^{s}} \partial_{u} \ln \left[u^{\nu} H_{\nu}^{(j)}(u)\right] \\
& +\frac{1}{2 \pi i} \sum_{j=1,2} \int_{C_{j}} \frac{d u}{u^{s}} \partial_{u} \ln \left[1-\frac{J_{\nu}(u) H_{\nu}^{(j)}(\eta u)}{H_{\nu}^{(j)}(u) J_{\nu}(\eta u)}\right],
\end{aligned}
$$

where $C_{1}$ and $C_{2}$ are the parts of the contour $C$ in upper and lower half-planes, $H_{\nu}^{(1,2)}(x)$ are the Hankel functions and $\eta$ is defined by (2.17). In the integrals over the imaginary axis we introduce the modified Bessel functions. Under the condition $\operatorname{Re} s>1$, the integrals over the circular parts of the contour $C$ tend to zero in the limit $R \rightarrow \infty$ and we obtain the integral representation of the function $\zeta_{p}(s)$. Substituting in (8.5), for the zeta function we get

$$
\begin{aligned}
\zeta(s)= & \frac{(D-1)(4 \pi)^{(1-D) / 2} \mu^{s+1}}{2 \Gamma(s / 2) \Gamma((D+1-s) / 2)} \int_{0}^{\infty} d x x^{D-1-s} \partial_{x} \\
& \times\left\{\ln \left[x^{-\nu} I_{\nu}\left(x z_{2}\right)\right]+\ln \left[x^{\nu} K_{\nu}\left(x z_{1}\right)\right]\right. \\
& \left.+\ln \left[1-\frac{I_{\nu}\left(x z_{1}\right) K_{\nu}\left(x z_{2}\right)}{K_{\nu}\left(x z_{1}\right) I_{\nu}\left(x z_{2}\right)}\right]\right\} .
\end{aligned}
$$

This integral representation is valid in the range $D<\operatorname{Re} s<D+1$. We need the analytical continuation of this expression to the point $s=-1$.

The part of the zeta function with the last term in figure braces of (8.13) is finite at $s=-1$. We will denote the corresponding contribution to the vacuum energy by $\Delta \mathcal{E}_{(\mathrm{II})}$. After integration by parts it is presented in the form

$$
\begin{aligned}
\Delta \mathcal{E}_{(\mathrm{II})}= & \frac{D-1}{(4 \pi)^{D / 2} \Gamma(D / 2)} \int_{0}^{\infty} d x x^{D-1} \\
& \times \ln \left[1-\frac{I_{\nu}\left(x z_{1}\right) K_{\nu}\left(x z_{2}\right)}{K_{\nu}\left(x z_{1}\right) I_{\nu}\left(x z_{2}\right)}\right] .
\end{aligned}
$$

Now, comparing with (7.6), we see that the Casimir interaction forces per unit surface acting on the plates are related to the energy $(8.14)$ by the formula

$$
f_{j}^{(\mathrm{int})}=-\left(z_{j} / \alpha\right)^{D+1} \frac{\partial}{\partial z_{j}} \Delta \mathcal{E}_{(\mathrm{II})} .
$$

This relation provides an alternative way for the evaluation of the Casimir forces acting on the plates. Note that the main part of previous investigations of the Casimir effect on the AdS bulk follows this procedure.

\section{B. Zeta function in the geometry with a single plate}

In the limit $z_{1} \rightarrow 0$ the part of the zeta function $\zeta(s)$ with the last two terms in figure braces of (8.13) tends to zero. Based on this, the part with the first term can be interpreted as the zeta function for the vacuum energy in the region $0 \leq z \leq z_{2}$ for the geometry of a single plate at $z=z_{2}$. This can also be seen by direct evaluation. Indeed, for a single plate at $z=z_{j}$ the mode functions for the vector potential in the region $0 \leq z \leq z_{j}$ have the form (2.6) with $C_{2}=0$. From the boundary conditions it follows that the eigenvalues of the quantum number $\lambda$ are solutions of the equation $J_{\nu}\left(\lambda z_{j}\right)=0$ with the same $\nu$ as in (2.12). The total energy of the vacuum per unit coordinate volume along the parallel directions is given by (8.3), with $z_{1}$ replaced by $z_{j}$, where 
now $\lambda_{\nu, n}$ is the $n$th positive zero of the function $J_{\nu}(x)$. Introducing the related zeta function $\zeta_{(\mathrm{I})}\left(s, z_{j}\right)$ similar to (8.4), by transformations like those presented above, the following integral representation is obtained:

$$
\begin{aligned}
\zeta_{(\mathrm{I})}\left(s, z_{j}\right)= & \frac{(D-1)(4 \pi)^{(1-D) / 2}\left(\mu z_{j}\right)^{s+1}}{2 \Gamma(s / 2) \Gamma((D+1-s) / 2) z_{j}^{D}} \\
& \times \int_{0}^{\infty} d x x^{D-1-s} \partial_{x} \ln \left[x^{-\nu} I_{\nu}(x)\right],
\end{aligned}
$$

with the range of validity $D<\operatorname{Re} s<D+1$.

The analytical continuation of (8.16) to the physical point $s=-1$ is done by the method widely discussed in the context of the Casimir effect. As the first step we decompose the integral into integrals over the regions $x \in[0,1]$ and $x \in[1, \infty)$. The part of the zeta function with the first integral is finite at $s=-1$. For the analytic continuation of the part with the second integral we subtract and add in the integrand the first $N$ terms of the corresponding asymptotic expansion for large values of $x$. This gives

$$
\begin{aligned}
\zeta_{(\mathrm{I})}\left(s, z_{j}\right)= & \frac{(D-1)(4 \pi)^{(1-D) / 2}\left(\mu z_{j}\right)^{s+1}}{2 \Gamma(s / 2) \Gamma((D+1-s) / 2) z_{j}^{D}} \\
& \times\left\{\int_{0}^{1} d x x^{D-1-s} \partial_{x} \ln \left[x^{-\nu} I_{\nu}(x)\right]\right. \\
& +\int_{1}^{\infty} d x x^{D-1-s}\left[\partial_{x} \ln \left(x^{-\nu} I_{\nu}(x)\right)-\sum_{k=0}^{N} \frac{b_{I, k}}{x^{k}}\right] \\
& \left.-\sum_{k=0}^{N} \frac{b_{I, k}}{D-s-k}\right\},
\end{aligned}
$$

where $b_{I, k}$ are the coefficients of the asymptotic expansion of the function $\partial_{x} \ln \left(x^{-\nu} I_{\nu}(x)\right)$ for large $x$. For those coefficients one has

$$
\begin{aligned}
& b_{I, 0}=1, \quad b_{I, 1}=-\nu-1 / 2, \\
& b_{I, k}=-(k-1) \beta_{k-1}, \quad k \geq 2 .
\end{aligned}
$$

Here, $\beta_{k}$ are defined by the relation

$$
\ln \left(\sum_{k=0}^{\infty} \frac{\alpha_{k}}{x^{k}}\right)=\sum_{k=1}^{\infty} \frac{\beta_{k}}{x^{k}}
$$

and

$$
\alpha_{k}=\frac{(-1)^{k} \Gamma(\nu+k+1 / 2)}{2^{k} k ! \Gamma(\nu-k+1 / 2)}
$$

are the coefficients of the asymptotic expansion of the function $\sqrt{2 \pi x} e^{-x} I_{\nu}(x)$ (the expressions for the first six coefficients $\beta_{k}$ are given, for example, in [7]).
For $N>D$, in (8.17) the integral over $[1, \infty)$ is finite at $s=-1$. The only singularity at this physical point comes from the simple pole corresponding to the term $k=D+1$ of the last sum in figure braces of (8.17). Denoting by $\zeta_{(\mathrm{I})}^{(p)}\left(s, z_{j}\right)$ and $\zeta_{(\mathrm{I})}^{(f)}\left(z_{j}\right)$ the pole and finite parts of the zeta function, respectively, from (8.17) one gets

$$
\zeta_{(\mathrm{I})}^{(p)}\left(s, z_{j}\right)=-\frac{(D-1) b_{I, D+1}}{D(4 \pi)^{D / 2} \Gamma(D / 2) z_{j}^{D}} \frac{1}{s+1} .
$$

The finite part of the zeta function gives the finite part of the vacuum energy induced by the brane $z=z_{j}$ in the region $0 \leq z \leq z_{j}$ :

$$
\begin{aligned}
\mathcal{E}_{(\mathrm{I})}^{(f)}\left(z_{j}\right)= & \zeta_{(\mathrm{I})}^{(f)}\left(z_{j}\right) \\
= & -\frac{(D-1) \pi^{-D / 2}}{2^{D} D \Gamma(D / 2) z_{j}^{D}}\left\{\int_{0}^{1} d x x^{D} \partial_{x} \ln \left(\frac{I_{\nu}(x)}{x^{\nu}}\right)\right. \\
& +\int_{1}^{\infty} d x x^{D}\left[\partial_{x} \ln \left(\frac{I_{\nu}(x)}{x^{\nu}}\right)-\sum_{k=0}^{N} \frac{b_{I, k}}{x^{k}}\right] \\
& +b_{I, D+1}\left[\ln \left(\mu z_{j}\right)-\psi\left(\frac{D}{2}+1\right)-\psi\left(-\frac{1}{2}\right)\right] \\
& \left.+\sum_{k=0}^{N} \frac{b_{I, k}}{k-D-1}\right\},
\end{aligned}
$$

where $\psi(x)=\Gamma^{\prime}(x) / \Gamma(x)$ is the digamma function and the prime on the summation sign means that the term $k=$ $D+1$ is excluded from the sum.

In order to provide a physical interpretation for the second term in figure braces of (8.13) let us consider the limit $z_{2} \rightarrow \infty$. The contributions with the first and third terms tend to zero. This allows us to interpret the function

$$
\begin{aligned}
\zeta_{(\mathrm{III})}\left(s, z_{j}\right)= & \frac{(D-1)(4 \pi)^{(1-D) / 2}\left(\mu z_{j}\right)^{s+1}}{2 \Gamma(s / 2) \Gamma((D+1-s) / 2) z_{j}^{D}} \\
& \times \int_{0}^{\infty} d x x^{D-1-s} \partial_{x} \ln \left[x^{\nu} K_{\nu}(x)\right]
\end{aligned}
$$

as the zeta function for the region $z_{j} \leq z<\infty$ in the geometry of a single plate at $z=z_{j}$. The validity range of this representation is the same as that for (8.16). The analytical continuation of the zeta function (8.23) is similar to that for the function (8.16). The corresponding pole part is presented as

$$
\zeta_{(\mathrm{III})}^{(p)}\left(s, z_{j}\right)=-\frac{(D-1) b_{K, D+1}}{D(4 \pi)^{D / 2} \Gamma(D / 2) z_{j}^{D}} \frac{1}{s+1} .
$$

The expression for the finite part of the vacuum energy, induced by the plate at $z=z_{j}$ in the region $z_{j} \leq z<\infty$, reads 


$$
\begin{aligned}
\mathcal{E}_{(\mathrm{III})}^{(f)}\left(z_{j}\right)= & \zeta_{(\mathrm{III})}^{(f)}\left(z_{j}\right) \\
= & -\frac{(D-1) \pi^{-D / 2}}{2^{D} D \Gamma(D / 2) z_{j}^{D}}\left\{\int_{0}^{1} d x x^{D} \partial_{x} \ln \left(x^{\nu} K_{\nu}(x)\right)\right. \\
& +\int_{1}^{\infty} d x x^{D}\left[\partial_{x} \ln \left(x^{\nu} K_{\nu}(x)\right)-\sum_{k=0}^{N} \frac{b_{K, k}}{x^{k}}\right] \\
& +b_{K, D+1}\left[\ln \left(\mu z_{j}\right)-\psi\left(\frac{D}{2}+1\right)-\psi\left(-\frac{1}{2}\right)\right] \\
& \left.+\sum_{k=0}^{N} \frac{b_{K, k}}{k-D-1}\right\},
\end{aligned}
$$

where

$$
\begin{aligned}
& b_{K, 0}=-1, \quad b_{K, 1}=\nu-1 / 2, \\
& b_{K, k}=(-1)^{k}(k-1) \beta_{k-1}, \quad k \geq 2 .
\end{aligned}
$$

In deriving (8.24) and (8.25) we have used the asymptotic expansion $\sqrt{2 x / \pi} e^{x} K_{\nu}(x)=\sum_{k=0}^{\infty}(-1)^{k} \alpha_{k} / x^{k}$ for the Macdonald function. Note that $b_{K, k}=-(-1)^{k} b_{I, k}$ for $k \geq 2$.

\section{Total zeta function and the vacuum energy}

Based on (8.21), (8.22), (8.24), and (8.25), for the pole part of the zeta function in the region between the plates we find

$$
\zeta^{(p)}(s)=\zeta_{(\mathrm{III})}^{(p)}\left(s, z_{1}\right)+\zeta_{(\mathrm{I})}^{(p)}\left(s, z_{2}\right)
$$

The pole terms in the right-hand side come from the single plate contributions. The finite part of the vacuum energy in the region between the plates is given by

$$
\mathcal{E}_{(\mathrm{II})}^{(f)}=\mathcal{E}_{(\mathrm{III})}^{(f)}\left(z_{1}\right)+\mathcal{E}_{(\mathrm{I})}^{(f)}\left(z_{2}\right)+\Delta \mathcal{E}_{(\mathrm{II})} .
$$

The divergent part of the vacuum energy coming from the pole term is absorbed by renormalizing the cosmological constants on the plates. Indeed, if $g_{(j) i k}$ is the induced metric tensor on the plate at $z=z_{j}$ and $R_{(j)}$ is the corresponding Ricci scalar, then the surface action for that plate is given by

$S_{(j)}=\frac{\left(\alpha / z_{j}\right)^{D}}{16 \pi G_{(j)}} \int d^{D} x d z \sqrt{\left|g_{(j)}\right|}\left[R_{(j)}-2 \Lambda_{(j)}\right] \delta\left(z-z_{j}\right)$,

where $\Lambda_{(j)}$ is the cosmological constant on the plate. As seen, the pole term in the vacuum energy has the same dependence on $z_{j}\left(z_{j}^{-D}\right)$ as the part in the action with the cosmological constant and can be absorbed by the renormalization of the latter. This point has been widely discussed in [6-9] in the context of Randall-Sundrum braneworlds.

Having the zeta functions in separate regions $0 \leq z \leq z_{1}$, $z_{1} \leq z \leq z_{2}$ and $z_{2} \leq z<\infty$ we can find the total zeta function:

$$
\begin{aligned}
\zeta_{\text {tot }}(s)= & \frac{(D-1)(4 \pi)^{(1-D) / 2} \mu^{s+1}}{2 \Gamma(s / 2) \Gamma((D+1-s) / 2)} \int_{0}^{\infty} d x x^{D-1-s} \partial_{x} \\
& \times\left\{\sum_{j=1,2} \ln \left[I_{\nu}\left(x z_{j}\right) K_{\nu}\left(x z_{j}\right)\right]\right. \\
& \left.+\ln \left[1-\frac{I_{\nu}\left(x z_{1}\right) K_{\nu}\left(x z_{2}\right)}{K_{\nu}\left(x z_{1}\right) I_{\nu}\left(x z_{2}\right)}\right]\right\} .
\end{aligned}
$$

The parts with the separate terms of the sum over $j$ are the total zeta functions in the problems with single plates at $z=z_{j}$. For the corresponding pole part one has

$\zeta_{\text {tot }}^{(p)}(s)=-\frac{1+(-1)^{D}}{s+1} \sum_{j=1,2} \frac{(D-1) b_{I, D+1}}{D(4 \pi)^{D / 2} \Gamma(D / 2) z_{j}^{D}}$,

where we have used the relation $b_{K, D+1}=(-1)^{D} b_{I, D+1}$. It is the sum of the pole parts for single plate geometries. For odd values of the spatial dimension $D$ the total pole part is zero and the finite part does not depend on the renormalization scale $\mu$. In this case the total vacuum energy is unambiguously defined and is given by

$$
\mathcal{E}=\sum_{j=1,2} \mathcal{E}_{j}+\Delta \mathcal{E}_{(\mathrm{II})},
$$

where

$$
\begin{aligned}
\mathcal{E}_{j}= & -\frac{(D-1) \pi^{-D / 2}}{2^{D} D \Gamma(D / 2) z_{j}^{D}}\left\{\frac{1}{D}-\sum_{l=1}^{N_{1}} \frac{4 l \beta_{2 l}}{2 l-D}\right. \\
& +\int_{0}^{1} d x x^{D} \partial_{x} \ln \left(I_{\nu}(x) K_{\nu}(x)\right) \\
& \left.+\int_{1}^{\infty} d x x^{D-1}\left[x \partial_{x} \ln \left(I_{\nu}(x) K_{\nu}(x)\right)+1+4 \sum_{l=1}^{N_{1}} \frac{l \beta_{2 l}}{x^{2 l}}\right]\right\},
\end{aligned}
$$

with $N_{1} \geq(D+1) / 2$, is the total vacuum energy for the geometry of a single boundary at $z=z_{j}$. For $D=3$ and $\nu=D / 2-1=1 / 2$ one finds

$$
\mathcal{E}_{j}=-\frac{\pi^{2}}{720 z_{j}^{3}},
$$

and for $D=3$ and $\nu=D / 2-2=-1 / 2$ we get 
TABLE I. The Casimir energies for a single plate in different numbers of odd spatial dimensions for the boundary conditions (2.4) and (2.5).

\begin{tabular}{lrrrr}
\hline \hline$D$ & 3 & 5 & \multicolumn{1}{c}{7} & \multicolumn{1}{c}{9} \\
\hline$z_{j}^{D} \mathcal{E}_{j}$, BC $(2.4)$ & -0.01371 & 0.01269 & -0.01640 & 0.02701 \\
$z_{j}^{D} \mathcal{E}_{j}$, BC $(2.5)$ & 0.01199 & -0.00410 & 0.00312 & -0.00378 \\
\hline \hline
\end{tabular}

$$
\mathcal{E}_{j}=\frac{7 \pi^{2}}{5760 z_{j}^{3}}
$$

In Table I we present the Casimir energy $\mathcal{E}_{j}$ in a single plate geometry for odd spatial dimensions and for boundary conditions (2.4) and (2.5). As seen, depending on the boundary condition and on the spatial dimension the energy can be either positive or negative.

The $z$ component of the force acting per unit surface of the plate at $z=z_{j}$ can be evaluated by using the formula

$$
f_{j}=-\left(z_{j} / \alpha\right)^{D+1} \frac{\partial}{\partial z_{j}} \mathcal{E}
$$

Based on the decomposition (8.32) of the vacuum energy the force is presented in the form (7.8), where the interaction part $f_{j}^{\text {(int) }}=(-1)^{j} p_{j}^{\text {(int) }}$ is obtained from the contribution $\Delta \mathcal{E}_{(\mathrm{II})}$ in (8.32) [see (8.15)]. The self-action part of the force comes from the single plate energies:

$$
f_{j}^{(\mathrm{s})}=-\left(z_{j} / \alpha\right)^{D+1} \frac{\partial}{\partial z_{j}} \mathcal{E}_{j}=\frac{D z_{j}^{D}}{\alpha^{D+1}} \mathcal{E}_{j} .
$$

Comparing with (8.33) we see that the self-action force does not depend on the location of the plate and it is the same for both plates. The corresponding values of $z_{j}^{D} \mathcal{E}_{j}$ for an odd number of spatial dimensions are given in Table I. The force acting on the plate is attractive with respect to the AdS boundary for $\mathcal{E}_{j}<0$ and repulsive for $\mathcal{E}_{j}>0$. It has opposite signs for the boundary conditions (2.4) and (2.5).

\section{APPLICATIONS TO $Z_{2}$-SYMMETRIC BRANEWORLD MODELS}

Higher-dimensional braneworld models of the RandallSundrum type [34] are among modern developments in theoretical physics where the AdS spacetime plays a central role. The original model with two branes is formulated on a slice of the $D=4$ AdS bulk with the extra dimension $x^{4}$ compactified on a $S^{1} / Z_{2}$ orbifold. The branes are located at the fixed points of the orbifold. Considering a general number of spatial dimensions $D$, the metric tensor in terms of the radial coordinate $y$ is given by $g_{\mu \nu}=$ $\operatorname{diag}\left(e^{-2|y| / \alpha} \eta_{i k},-1\right)$ with the fixed points $y=0$ and $y=a$ and with $-a \leq y \leq a$. The two regions $y<0$ and $y>0$ are identified by the $Z_{2}$ symmetry. The boundary conditions for fields propagating in the bulk are dictated by that symmetry (see, for example, the discussion in $[35,36]$ for the case $D=4$ ).

In fact, one has two reflection symmetries. The first one corresponds to the reflection with respect to the brane at $y=0$ with $y \rightarrow-y$ and the second one is the symmetry under the reflection with respect to the brane at $y=a$ with $y-a \rightarrow a-y$. For fields even under both these reflections the mode functions are given by (2.6) with $z=\alpha e^{|y| / \alpha}$. The corresponding boundary conditions are obtained by the integration of the field equation near the points $y=0$ and $y=a$. The conditions on the radial function are reduced to the constraints $\partial_{D} A_{(\beta) l}=0$ for $y=0, a$ and correspond to the boundary condition (2.5) discussed in previous sections. For odd fields the modes are obtained from (2.6) taking $z=\alpha e^{|y| / \alpha}$ and adding an additional coefficient $\operatorname{sgn}(y)$. In this case from the continuity of the modes at the locations of the branes we get the boundary conditions $A_{(\beta) l}=0$ for $y=0, a$ that correspond to the condition (2.4). From this consideration it follows that the modes for massless vector fields in higher-dimensional generalization of the Randall-Sundrum models are given by (2.10) with $\nu=D / 2-1$ for odd fields under the $Z_{2}$ symmetry and $\nu=D / 2-2$ for even fields. The normalization constants in both these cases are given by (2.16) with an additional factor $1 / 2$ in the right-hand side. The latter difference is related to the fact that now the integration over $y$ in the normalization condition (2.7) goes over the region $[-a, a]$ instead of $[0, a]$ in the problem we have discussed before. Hence, the expressions for the local characteristics of the electromagnetic vacuum in $Z_{2}$-symmetric braneworlds, such as the correlators, the VEVs of the fields squared, of the energy-momentum tensor and the Casimir forces, are obtained from the expressions given above with an additional factor $1 / 2$ and with $z_{1}=\alpha, z_{2}=\alpha e^{a / \alpha}$. For odd and even fields with respect to the $Z_{2}$ reflection the results for the boundary conditions (2.4) and (2.5) have to be taken. The expression for the total Casimir energy in the region between the branes remains the same (the integration over the region $y \in[-a, a]$ brings a factor of 2). Regions I and III that we have discussed above are excluded in the braneworld setup.

We can also consider the case when the $Z_{2}$ parities of the field with respect to the branes $y=0$ and $y=a$ have opposite signs (for different combinations of parities in the case of vector fields see [36]). For example, let us discuss the field odd under the reflection with respect to the brane $y=0$ and even under the reflection with respect to the second brane at $y=a$. Now, the field obeys the boundary condition $A_{(\beta) l}=0$ for $y=0$ and the condition $\partial_{D} A_{(\beta) l}=0$ for $y=a$. In the region $0 \leq y \leq a$ the mode functions are given by (2.6). From the boundary condition at $y=0$ it follows that $c_{2} / c_{1}=-J_{D / 2-1}\left(\lambda z_{1}\right) / Y_{D / 2-1}\left(\lambda z_{1}\right)$. For the corresponding modes we get 


$$
A_{(\beta) \mu}=C \epsilon_{(\sigma) \mu} z^{D / 2-1} g_{D / 2-1, D / 2-1}\left(\lambda z_{1}, \lambda z\right) e^{i k_{l} x^{l}} .
$$

The boundary condition on the brane $y=a$ leads to the equation $g_{D / 2-1, D / 2-2}\left(\lambda z_{1}, \lambda z_{2}\right)=0$ for the eigenvalues of the quantum number $\lambda$. The normalization coefficient is obtained from (2.7) with the integration over the region $y \in[-a, a]$ and is given by

$$
|C|^{2}=\frac{\pi^{2} \alpha^{3-D} \lambda_{n}^{2}}{4(2 \pi)^{D-2} \omega z_{1}^{2}}\left[\frac{J_{D / 2-1}^{2}\left(\lambda_{n}\right)}{J_{D / 2-2}^{2}\left(\lambda_{n} \eta\right)}-1\right]^{-1},
$$

with $\lambda_{n}$ being the eigenvalues for $\lambda z_{1}$. The investigation of the VEVs is similar to that we have described in the previous sections. The summation formula for the series in the mode sums over the eigenvalues of $\lambda$ is given in [26,27]. Note that we could consider the analog of the problems discussed in the previous sections with the boundary condition (2.4) on the plate $z=z_{1}$ and the condition (2.5) on the plate $z=z_{2}$. The corresponding mode functions in the region $z_{1} \leq z \leq z_{2}$ are given by (9.1) with an additional factor 2 in the expression (9.2) for the normalization coefficient.

\section{SUMMARY}

We have discussed the effects of two parallel plates in AdS spacetime on the properties of the electromagnetic vacuum in an arbitrary number of spatial dimensions. The plates are parallel to the AdS boundary and two types of boundary conditions were discussed on them. The first one is the generalization of the perfect conductor boundary condition for an arbitrary number of spatial dimensions and the second one corresponds to the confining boundary condition used in quantum chromodynamics to confine gluons. In the model under consideration the properties of the electromagnetic vacuum are encoded in two-point functions and we have started the consideration from the two-point function of the vector potential. It is presented in the form of the sum over a complete set of electromagnetic modes. In the region between the plates, the eigenvalues of the quantum number corresponding to the direction normal to the plates are roots of Eq. (2.12). The application of the summation formula (3.5) to the corresponding series allowed us to extract the contribution of the single plate and to present the second-plate-induced part in the form that is well adapted for the evaluation of the VEVs for local observables. Two equivalent representations of the twopoint function for the vector potential are given by (3.9) and (3.16). The VEVs of local physical observables are obtained from the two-point functions for the field tensor and the expressions for those functions are given in Sec. IV. We have provided the expressions for both the single-plateand second-plate-induced contributions.

As important local characteristics of the vacuum state we have considered the VEVs of the electric and magnetic fields squared and the photon condensate. The single-plate and second-plate-induced contributions to the VEV of the electric field square are positive for the boundary condition (2.4) and negative for the condition (2.5). The signs of the corresponding contributions in the VEV of the magnetic field squared and in the photon condensate are opposite to that for the electric field. Near the plates the VEVs are dominated by single plate contributions. In those regions the effects of the gravity on the VEVs are weak and the leading terms in the asymptotic expansions over the distance from the plate coincide with those in the corresponding problem on the Minkowski bulk. The effects of the gravity are essential for separations between the plates larger than the curvature radius of the background geometry. Having the correlators for the field tensor one can evaluate the Casimir-Polder forces acting on a polarizable particle. As an illustration we have considered the simplest case of isotropic polarizability in the static limit. Near the plate, the Casimir-Polder force is attractive (repulsive) with respect to that plate for the boundary condition (2.4) [(2.5)].

Similar investigations for the VEV of the energymomentum tensor are presented in Sec. VI. The offdiagonal components vanish and the diagonal components are decomposed as (6.2) and (6.3), with the single plate contributions given by (6.4). The vacuum stresses along the directions parallel to the plates are equal to the energy density. We have checked that the boundary-induced VEV in the energy-momentum tensor obeys the covariant continuity equation and its trace is expressed in terms of the photon condensate as (6.6). For $D \geq 4$ the boundaryinduced contribution in the vacuum energy density is negative for the condition (2.4) and positive for (2.5). In $D=3$ spatial dimensions the electromagnetic field is conformally invariant and the vacuum energy-momentum tensor in region II is given by simple expressions (6.8). The latter is the same for the boundary conditions (2.4) and (2.5). For $D=3$ the boundary-induced VEV in the energymomentum tensor vanishes in region III. In region I the vacuum energy-momentum tensor is given by (6.9) for the condition (2.4) and by (6.10) for (2.5). Those expressions are different and that is a consequence of different interactions with the AdS boundary.

Based on the expressions for the normal stress we have investigated the Casimir forces acting on the plates. They are decomposed into the self-action and interaction contributions. The first parts come from the single plate contributions in the normal stress and for $D \neq 3$ require an additional renormalization because of the surface divergences. The interaction parts in the vacuum pressures on the plates are given by (7.4) and they are attractive for both the boundary conditions (2.4) and (2.5). An alternative expression is given by (7.6). For $D=3$ the self-action forces are finite and the total forces per unit surface of the plates are presented as (7.9) and (7.10). The force on the right plate is directed toward the AdS boundary for both the 
boundary conditions. The force on the left plate for the condition (2.5) is directed toward the AdS horizon. For the condition (2.4) the force on the left plate is directed toward the AdS horizon for small separations and toward the AdS boundary for large separations. For general $D$ and at small separations between the plates, compared to the AdS curvature radius, the leading terms in the Casimir forces coincide with that for the plates in the Minkowski bulk. This is a consequence of the fact that at small separations the contribution of the vacuum fluctuations with small wavelengths dominates in the vacuum forces and the influence of gravity on those fluctuations is weak. At separations larger than the AdS curvature scale the gravity essentially changes the behavior of the Casimir forces: considered as functions of the interplate separation, the forces decay exponentially in contrast to the power-law decay for the Minkowski bulk. Another feature differing the AdS and Minkowski bulks is that the forces on the left and right plates differ.

Among the interesting directions in the investigations of the Casimir effect is the relation between the local and global characteristics of the vacuum state. In Sec. VIII, the total vacuum energy per unit surface of the plate is considered. For regularization of the corresponding divergent expression we have used the generalized zeta function approach. By using the standard technique, an integral representation of the zeta function is provided for the region between the plates with separated single plate and interaction contributions. The latter is finite at the physical point and the analytic continuation is required for single plate parts only. In order to do that we have employed the wellknown procedure from the theory of the Casimir effect. The pole and finite parts of the zeta functions are explicitly provided for both regions in the geometry of a single plate.
The pole parts can be absorbed by the renormalization of the cosmological constant on the plate. Considering the total zeta function for a single plate as the sum of the zeta functions in separate regions, the pole parts are canceled in odd numbers of spatial dimensions and the total vacuum energy, given by (8.33), does not depend on the mass scale in the renormalization procedure. For a single plate, the force per unit surface of the plate is obtained by differentiation of the Casimir energy and does not depend on the location of the plate. Depending on the boundary condition and on the spatial dimension, that force can be either attractive or repulsive with respect to the AdS boundary (see the numerical data in Table I).

The results obtained can be directly used for the investigation of quantum vacuum effects in $Z_{2}$-symmetric braneworlds of the Randall-Sundrum type. The boundary conditions on the branes are dictated by the $Z_{2}$ symmetry and are reduced to the condition (2.4) for odd fields and to the condition (2.5) for even fields. The expressions for both the local and global characteristics of the vacuum state are obtained from those we have discussed. One can consider also the situation when the $Z_{2}$ parities of the fields on the branes are different. In this case we have the condition (2.4) on one brane and the condition (2.5) on the other. The corresponding mode functions are given by (9.1) with the normalization constant (9.2). The evaluation procedure for the VEVs is similar to that we have described in this paper.

\section{ACKNOWLEDGMENTS}

A. S. K. and H. G. S. were supported by Grant No. 18T1C355 of the Committee of Science of the Ministry of Education, Science, Culture and Sport RA.
[1] V. M. Mostepanenko and N. N. Trunov, The Casimir Effect and Its Applications (Clarendon, Oxford, 1997); K. A. Milton, The Casimir Effect: Physical Manifestation of Zero-Point Energy (World Scientific, Singapore, 2002); V. A. Parsegian, Van der Waals Forces: A Handbookfor Biologists, Chemists, Engineers, and Physicists (Cambridge University Press, Cambridge, England, 2005); M. Bordag, G. L. Klimchitskaya, U. Mohideen, and V. M. Mostepanenko, Advances in the Casimir Effect (Oxford University Press, New York, 2009); Casimir Physics, edited by D. Dalvit, P. Milonni, D. Roberts, and F. da Rosa, Lecture Notes in Physics Vol. 834 (SpringerVerlag, Berlin, 2011).

[2] C. Callan and F. Wilezek, Nucl. Phys. B340, 366 (1990); E. Kiritsis and C. Kounnas, Nucl. Phys. B, Proc. Suppl. 41, 331 (1995).

[3] O. Aharony, S. S. Gubser, J. Maldacena, H. Ooguri, and Y. Oz, Phys. Rep. 323, 183 (2000); H. Năstase, Introduction to
AdS/CFT Correspondence (Cambridge University Press, Cambridge, England, 2015); M. Ammon and J. Erdmenger, Gauge/Gravity Duality: Foundations and Applications (Cambridge University Press, Cambridge, England, 2015).

[4] A.S.T. Pires, AdS/CFT Correspondence in Condensed Matter (Morgan \& Claypool, San Rafael, CA, 2014); J. Zaanen, Y.-W. Sun, Y. Liu, and K. Schalm, Holographic Duality in Condensed Matter Physics (Cambridge University Press, Cambridge, England, 2015); R.-G. Cai, L. Li, L.-F. Li, and R.-Q. Yang, Sci. China-Phys. Mech. Astron. 58, 060401 (2015); E. Kiritsis and L. Li, J. High Energy Phys. 01 (2016) 147.

[5] R. Maartens and K. Koyama, Living Rev. Relativity 13, 1 (2010).

[6] M. Fabinger and P. Horava, Nucl. Phys. B580, 243 (2000); S. Nojiri, S. Odintsov, and S. Zerbini, Phys. Rev. D 62 , 064006 (2000); S. Nojiri, O. Obregon, and S. Odintsov, 
Phys. Rev. D 62, 104003 (2000); D. J. Toms, Phys. Lett. B 484, 149 (2000); W. D. Goldberger and I. Z. Rothstein, Phys. Lett. B 491, 339 (2000); S. Nojiri and S. Odintsov, J. High Energy Phys. 07 (2000) 049; J. Garriga, O. Pujolàs, and T. Tanaka, Nucl. Phys. B605, 192 (2001); I. H. Brevik, K. A. Milton, S. Nojiri, and S. D. Odintsov, Nucl. Phys. B599, 305 (2001); A. A. Saharian and M. R. Setare, Phys. Lett. B 552, 119 (2003); E. Elizalde, S. Nojiri, S. D. Odintsov, and S. Ogushi, Phys. Rev. D 67, 063515 (2003); R. Durrer and M. Ruser, Phys. Rev. Lett. 99, 071601 (2007); M. Ruser and R. Durrer, Phys. Rev. D 76, 104014 (2007); A. A. Saharian and A. L. Mkhitaryan, J. High Energy Phys. 082007 063; M. Frank, I. Turan, and L. Ziegler, Phys. Rev. D 76, 015008 (2007); A. Flachi and T. Tanaka, Phys. Rev. D 80, 124022 (2009); L. P. Teo, Phys. Lett. B 682, 259 (2009); M. Rypestol and I. Brevik, New J. Phys. 12, 013022 (2010); R. Obousy and G. Cleaver, J. Geom. Phys. 61, 577 (2011); L. P. Teo, Int. J. Mod. Phys. A 28, 1350158 (2013); E. R. Bezerra de Mello, A. A. Saharian, and M. R. Setare, Phys. Rev. D 92, 104005 (2015); N. Haba and T. Yamada, arXiv:1903.10160.

[7] A. Flachi and D. J. Toms, Nucl. Phys. B610, 144 (2001).

[8] A. Flachi, I. G. Moss, and D. J. Toms, Phys. Lett. B 518, 153 (2001); A. Flachi, I. G. Moss, and D. J. Toms, Phys. Rev. D 64, 105029 (2001); K. Uzawa, Prog. Theor. Phys. 110, 457 (2003).

[9] J. Garriga and A. Pomarol, Phys. Lett. B 560, 91 (2003); N. Haba and T. Yamada, arXiv:1903.10160.

[10] L. P. Teo, J. High Energy Phys. 10 (2010) 019.

[11] T. Nishioka, S. Ryu, and T. Takayanagi, J. Phys. A 42, 504008 (2009); T. Takayanagi, Phys. Rev. Lett. 107, 101602 (2011); M. Fujita, T. Takayanagi, and E. Tonni, J. High Energy Phys. 11 (2011) 043.

[12] R. A. Knapman and D. J. Toms, Phys. Rev. D 69, 044023 (2004); A. A. Saharian, Nucl. Phys. B712, 196 (2005); S.-H. Shao, P. Chen, and J.-A. Gu, Phys. Rev. D 81, 084036 (2010); E. Elizalde, S. D. Odintsov, and A. A. Saharian, Phys. Rev. D 87, 084003 (2013).

[13] A. Flachi, J. Garriga, O. Pujolàs, and T. Tanaka, J. High Energy Phys. 08 (2003) 053; A. Flachi and O. Pujolàs, Phys. Rev. D 68, 025023 (2003); A. A. Saharian, Phys. Rev. D 73, 044012 (2006); 73, 064019 (2006); 74, 124009 (2006); E. Elizalde, M. Minamitsuji, and W. Naylor, Phys. Rev. D 75, 064032 (2007); R. Linares, H. A. MoralesTécotl, and O. Pedraza, Phys. Rev. D 77, 066012 (2008); M. Frank, N. Saad, and I. Turan, Phys. Rev. D 78, 055014 (2008).

[14] E. R. Bezerra de Mello, A. A. Saharian, and V. Vardanyan, Phys. Lett. B 741, 155 (2015); S. Bellucci, A. A. Saharian, and V. Vardanyan, Phys. Rev. D 96, 065025 (2017); S. Bellucci, A. A. Saharian, and V. Vardanyan, J. High Energy Phys. 11 (2015) 092; S. Bellucci, A. A. Saharian, and V. Vardanyan, Phys. Rev. D 93, 084011 (2016); S. Bellucci, A. A. Saharian, D. H. Simonyan, and V. Vardanyan, Phys. Rev. D 98, 085020 (2018); S. Bellucci, A. A. Saharian, H. G. Sargsyan, and V. V. Vardanyan, Phys. Rev. D 101, 045020 (2020).

[15] S. Nojiri and S. Odintsov, Phys. Lett. B 484, 119 (2000); W. Naylor and M. Sasaki, Phys. Lett. B 542, 289 (2002);
E. Elizalde, S. Nojiri, S. D. Odintsov, and S. Ogushi, Phys. Rev. D 67, 063515 (2003); I. G. Moss, W. Naylor, W. Santiago-Germán, and M. Sasaki, Phys. Rev. D 67, 125010 (2003); O. Pujolàs and T. Tanaka, J. Cosmol. Astropart. Phys. 12 (2004) 009; A. Flachi, A. Knapman, W. Naylor, and M. Sasaki, Phys. Rev. D 70, 124011 (2004); J. P. Norman, Phys. Rev. D 69, 125015 (2004); W. Naylor and M. Sasaki, Prog. Theor. Phys. 113, 535 (2005); O. Pujolàs and M. Sasaki, J. Cosmol. Astropart. Phys. 09 (2005) 002.

[16] B. Allen and T. Jacobson, Commun. Math. Phys. 103, 669 (1986); H. Janssen and C. Dullemond, J. Math. Phys. (N.Y.) 28, 1023 (1987); N. C. Tsamis and R. P. Woodard, J. Math. Phys. (N.Y.) 48, 052306 (2007); M. B. Fröb and A. Higuchi, J. Math. Phys. (N.Y.) 55, 062301 (2014); A. Belokogne, A. Folacci, and J. Queva, Phys. Rev. D 94, 105028 (2016).

[17] A. Ishibashi and R. M. Wald, Classical Quantum Gravity 21, 2981 (2004); D. Marolf and S. F. Ross, J. High Energy Phys. 11 (2006) 085; O. Aharony, M. Berkooz, D. Tong, and Sh. Yankielowicz, J. High Energy Phys. 02 (2013) 076.

[18] H. Davoudiasl, J. L. Hewett, and T. G. Rizzo, Phys. Lett. B 473, 43 (2000); A. Pomarol, Phys. Rev. Lett. 85, 4004 (2000); L. Randall and M. D. Schwartz, J. High Energy Phys. 11 (2001) 003.

[19] A. S. Kotanjyan, A. A. Saharian, H. G. Sargsyan, and D. H. Simonyan, Proc. Sci., MPCS2015 (2015) 021.

[20] A. A. Saharian, A. S. Kotanjyan, and A. A. Saharyan, Phys. Math. Sci. 3, 37 (2016), http://ysu.am/science/en/ 1478069496.

[21] A. S. Kotanjyan and A. A. Saharian, Phys. At. Nucl. 80, 562 (2017).

[22] A. S. Kotanjyan, A. A. Saharian, and A. A. Saharyan, Galaxies 5, 102 (2017).

[23] A. A. Saharian, A. S. Kotanjyan, and H. A. Nersisyan, Phys. Lett. B 728, 141 (2014).

[24] A. S. Kotanjyan, A. A. Saharian, and H. A. Nersisyan, Phys. Scr. 90, 065304 (2015).

[25] S. Bellucci and A. A. Saharian, Phys. Rev. D 88, 064034 (2013).

[26] A. A. Saharian, Izvestiia Akademii nauk Armianskoi SSR Matematika 22, 166 (1987) [Sov. J. Contemp. Math. Anal. 22, 70 (1987)].

[27] A. A. Saharian, The Generalized Abel-Plana Formula with Applications to Bessel Functions and Casimir Effect (Yerevan State University, Yerevan, 2008); The Abdus Salam International Centre for Theoretical Physics, Report No. ICTP/2007/082; arXiv:0708.1187.

[28] A. Vainshtein and V. Zakharov, Phys. Lett. B 225, 415 (1989).

[29] A. A. Saharian, A. S. Kotanjyan, A. A. Saharyan, and H. G. Sargsyan, Int. J. Mod. Phys. A 35, 2040029 (2020).

[30] A. Romeo and A. A. Saharian, J. Phys. A 35, 1297 (2002).

[31] A. A. Saharian, Phys. Rev. D 69, 085005 (2004).

[32] A. A. Saharian, Phys. Rev. D 70, 064026 (2004); 74, 124009 (2006); A. A. Saharian and H. G. Sargsyan, Astrophys. 61, 375 (2018).

[33] E. Elizalde, S. D. Odintsov, A. Romeo, A. A. Bytsenko, and S. Zerbini, Zeta Regularization Techniques with 
Applications (World Scientific, Singapore, 1994); K. Kirsten, Spectral Functions in Mathematics and Physics (CRC Press, Boca Raton, FL, 2001); A. A. Bytsenko, G. Cognola, E. Elizalde, V. Moretti, and S. Zerbini, Analytic Aspects of Quantum Fields (World Scientific, Singapore, 2003).
[34] L. Randall and R. Sundrum, Phys. Rev. Lett. 83, 3370 (1999); L. Randall and R. Sundrum, Phys. Rev. Lett. 83, 4690 (1999).

[35] T. Gherghetta and A. Pomarol, Nucl. Phys. B586, 141 (2000).

[36] S. Chan, S. Ch. Park, and J. Song, Phys. Rev. D 71, 106004 (2005). 